\title{
SIMPLE SUBGROUPS OF LARGE RANK IN GROUPS OF LIE TYPE
}

\author{
MARTIN W. LIEBECK, JAN SAXL, and DONNA M. TESTERMAN
}

[Received 4 November 1993-Revised 17 January 1995]

\section{Introduction}

Let $X$ and $G$ be simple, connected, linear algebraic groups over an algebraically closed field $k$ of characteristic $p$ (possibly $p=0$ ), and suppose that $\operatorname{rank}(X)>$ $\frac{1}{2} \operatorname{rank}(G)$. In this paper we determine all closed, connected subgroups of $G$ which are isomorphic to $X$. We also prove a corresponding result for finite groups of Lie type. It turns out that there are just finitely many conjugacy classes of such subgroups $X$ in $G$, except when $X=D_{4}, G=E_{7}$ and $p=2$; in this case there are infinitely many classes.

Many of our subgroups $X$ contain long root subgroups of $G$. Arbitrary such simple subgroups of $G$ are classified in [25, Theorem 2.1], and we make extensive use of the results and methods of [25] in our proofs. For finite groups, some very special cases of our results can be found in [22].

Our result for connected subgroups $X$ (Theorem 1) has some overlap with recent work [26] of the first author and G. Seitz, in which arbitrary simple connected subgroups of simple algebraic groups of exceptional type are determined, assuming some mild restrictions on the characteristic of the field $k$. In Theorem 1, we make no assumption on the characteristic, and we also cover classical types; moreover, Theorem 1 is required for the proof of our result on finite groups (Theorem 2). As a consequence of Theorem 2, we determine all almost simple maximal subgroups of finite exceptional groups satisfying the condition on ranks (Theorem 3).

To state our results we require a definition taken from [25]. A subsystem subgroup of $G$ is a simple, closed, connected subgroup $Y$ which is normalized by a maximal torus of $G$. Let $\Sigma(G)$ be the root system of $G$, and for $\alpha \in \Sigma(G)$, let $U_{\alpha}$ denote the $T$-root subgroup corresponding to $\alpha$, where $T$ is a fixed maximal torus normalizing $Y$. Then $Y=\left\langle U_{\alpha} \mid \alpha \in \Sigma_{0}\right\rangle$, where either $\Sigma_{0}$ is a closed subsystem of $\Sigma(G)$ or $(G, p)$ is $\left(B_{n}, 2\right),\left(C_{n}, 2\right),\left(F_{4}, 2\right)$ or $\left(G_{2}, 3\right)$ and $\Sigma_{0}$ lies in the dual of a closed subsystem. The subsystem subgroups of $G$ are well known and can be listed by an application of the result in [6]. We can now state our result for algebraic groups. Throughout, by a simple algebraic group we shall mean a simple connected linear algebraic group.

THEOREM 1. Let $G$ be a simple algebraic group over an algebraically closed field $k$ of characteristic $p$. Suppose that $X$ is a simple, closed, connected subgroup of $G$ such that $\operatorname{rank}(X)>\frac{1}{2} \operatorname{rank}(G)$. Then there is a subsystem subgroup $Y$ of $G$ such that $X \leqslant Y$ and one of the following holds:

(I) $X=Y$; 
(II) $X=Y_{\tau}$, where $\tau$ is a graph automorphism of $Y$ of order 2;

(III) $X, Y$ are as in Table 1;

(IV) $X=D_{4}, p=2$ and $Y=G=E_{7}$; there are infinitely many $G$-conjugacy classes of subgroups $D_{4}$ in $G$, all lying in an $E_{6}$-parabolic.

Each possibility for $X$ in Table 1 corresponds to a unique conjugacy class of subgroups in G. Furthermore, no subgroup $X$ in Table 1 satisfies (I) or (II).

TABLE 1

\begin{tabular}{lll}
\hline$X$ & \multicolumn{1}{c}{$Y$} & \multicolumn{1}{c}{ Conditions } \\
\hline$D_{n}$ & $A_{2 n-1}$ & $Y=G, p=2$ \\
$G_{2}$ & $B_{3}$ or $C_{3}$ & $Y=G, p=2$ if $G=C_{3}$ \\
$A_{2}$ & $B_{3}$ & $Y=G, p=3, X$ irreducible in $G$ \\
$C_{4}$ & $E_{6}$ & $p=2, X<F_{4}<Y, G=E_{6}$ or $E_{7}$ \\
$D_{4}$ & $E_{6}$ & $p=2, X<F_{4}<Y=G$ with $X$ generated by short root subgroups of $F_{4}$ \\
\hline
\end{tabular}

Remarks. (1) The pairs $(X, Y)$ occurring in (II) are $\left(C_{n}, A_{2 n-1}\right),\left(B_{n-1}, D_{n}\right)$ and $\left(F_{4}, E_{6}\right)$, and for $p \neq 2,\left(D_{n}, A_{2 n-1}\right)$ and $\left(C_{4}, E_{6}\right)$.

(2) In (IV), the projection of $X=D_{4}$ in the Levi factor $E_{6}$ lies in a subgroup $F_{4}$, and is generated by short root subgroups of this $F_{4}$. An explicit parameterization of the conjugacy classes of subgroups $D_{4}$ in this case is given in Remark 2.9. All the subgroups have the same composition factors on $L\left(E_{7}\right)$; this is in striking contrast with [26, Theorem 4], which states that under certain assumptions on the characteristic, simple closed connected subgroups of exceptional algebraic groups $G$ are determined up to conjugacy by their composition factors on $L(G)$.

(3) It seems likely that a result can be obtained with the hypothesis $\operatorname{rank}(X)>\frac{1}{2} \operatorname{rank}(G)$ relaxed; indeed, this has been done in [26] for exceptional groups $G$, under mild characteristic assumptions. However, for classical groups $G$, even when $\operatorname{rank}(X)=\frac{1}{2} \operatorname{rank}(G)$, representation-theoretic complications arise which are not present in our proof.

We now come to our results on finite groups. If $X$ is a finite group of Lie type over $\mathbb{F}_{q}$, where $q=p^{a}$ ( $p$ prime), then $X=O^{p^{\prime}}\left(\bar{X}_{\delta}\right)$, where $\bar{X}$ is a simple algebraic group over an algebraically closed field of characteristic $p$ and $\delta$ is a suitable Frobenius morphism of $\bar{X}$; we call $\operatorname{rank}(\bar{X})$ the untwisted rank of $X$.

THEOREM 2. Let $G$ be as in Theorem 1, and let $X=X(q)$ be a finite quasisimple group of Lie type over $\mathbb{F}_{q}$, where $q=p^{a}>2$, such that $X<G$ and the untwisted rank l of $X$ is more than $\frac{1}{2} \operatorname{rank}(G)$. Then one of the following holds:

(I) there is a simple, closed, connected subgroup $\bar{X}$ of $G$ of rank $l$ such that $X=O^{p^{\prime}}\left(\bar{X}_{\delta}\right)$ for some Frobenius morphism $\delta$ of $\bar{X}$; moreover, if $X<G_{\sigma}$ for some Frobenius morphism $\sigma$ of $G$, then $\bar{X}$ is $\sigma$-stable;

(II) $p=3, X=B_{2}(3)$ or ${ }^{2} A_{2}(3)$, and $G=B_{3}$ or $C_{3}$ respectively (with $X$ indecomposable on the natural 7- or 6-dimensional module for $G$ );

(III) $G=E_{8}$ and $X={ }^{2} A_{5}(5)$ or ${ }^{2} D_{5}(3)$. 
REMARKS. (1) In both cases of (II), there is a unique conjugacy class of such subgroups $X$ in $G$; in (III), we have not determined the conjugacy classes of subgroups $X$.

(2) In (I), the subgroup $\bar{X}$ of $G$ is of course identified by Theorem 1 .

(3) Theorem 2 can be used to determine the $G_{\sigma}$-classes of quasisimple subgroups $X$ of $G_{\sigma}$ with untwisted rank bigger than $\frac{1}{2} \operatorname{rank}(G)$, as follows. The $G$-classes of subgroups $\bar{X}$ (as in Theorem 2) are given by Theorem 1 . Then the $G_{\sigma}$-classes of such $\bar{X}$ are given by an application of Lang's theorem (cf. [10, Proposition 1]). Finally, the classes of subgroups $X$ in $\bar{X}$ are given by $[25,5.1]$. (Note that if $X$ is $G_{\sigma}$-conjugate to $Y$ then $\bar{X}$ is $G_{\sigma}$-conjugate to $\bar{Y}$.)

Our final theorem is a consequence of Theorems 1 and 2 for maximal subgroups of the finite exceptional groups of Lie type. The reduction theorem in [24, Theorem 2] determines all maximal subgroups of such groups which are not almost simple; Theorem 3 below is a contribution to the remaining case, and determines the almost simple subgroups which satisfy a suitable rank condition.

In the statement we use the following notation. Let $G$ be a simple adjoint algebraic group of exceptional type over an algebraically closed field $k$ of characteristic $p>0$, and let $\sigma$ be a Frobenius morphism of $G$, so that $L=O^{p^{\prime}}\left(G_{\sigma}\right)$ is a finite simple group of exceptional Lie type over $k_{0}$, a finite field of characteristic $p$. Let $L_{1}$ be an almost simple group with socle $L$. A simple subgroup of $L$ of the same type as $L$ (possibly twisted) over a subfield of $k_{0}$ will be called a subfield or twisted subgroup of $L$; these subgroups are determined up to conjugacy by $[25,5.1]$. Also, a subgroup of $L_{1}$ of the form $N_{L_{1}}\left(D_{\sigma}\right)$, where $D$ is a $\sigma$-stable reductive subgroup of $G$ containing a maximal torus, is called a subgroup of maximal rank; the subgroups of maximal rank which are maximal in $L_{1}$ are determined in [23].

THEOREM 3. Let $L_{1}$ be an almost simple group with socle $L=O^{p^{\prime}}\left(G_{\sigma}\right)$ as above, and let $M$ be an almost simple maximal subgroup of $L_{1}$. Suppose that $F^{*}(M)=$ $X(q)$, a simple group of Lie type over $\mathbb{F}_{q}$, where $q=p^{a}>2$ and the untwisted rank of $X(q)$ is more than $\frac{1}{2} \operatorname{rank}(G)$; assume also that $(G, X(q))$ is not $\left(E_{8},{ }^{2} A_{5}(5)\right)$ or $\left(E_{8},{ }^{2} D_{5}(3)\right)$. Then one of the following holds:

(i) $M$ is a subgroup of maximal rank;

(ii) $X(q)$ is a subfield or twisted subgroup;

(iii) $L=E_{6}^{\varepsilon}(q)$ and $X(q)=C_{4}(q)(q$ odd $)$ or $F_{4}(q)$.

Notation. Throughout the paper, $k$ denotes an algebraically closed field of characteristic $p$. If $X$ is a connected reductive group over $k$ and $\lambda$ a dominant weight with respect to a fixed base of the root system of $X$, we let $V_{X}(\lambda)$ and $W_{X}(\lambda)$ denote the rational irreducible $k X$-module with high weight $\lambda$ and the Weyl module with high weight $\lambda$, respectively. For a weight $\mu<\lambda$, we will write $V_{X}(\lambda)_{\mu}$ for the $T$-weight space corresponding to the weight $\mu$. The same notation will be used if $X$ is finite, where we consider the module as the restriction of a module for the corresponding simple algebraic group. If $V$ is a $k X$-module and $\tau$ is an automorphism of $X$ (as abstract group) which is also a morphism if $X$ is connected, we denote by $V^{\tau}$ the $k X$-module obtained from $V$ by twisting the action of $X$ by $\tau$. And if $\tau$ is a $p^{e}$-power field automorphism, we write $V^{\tau}=V^{(e)}$.

If $V_{1}, V_{2}, \ldots, V_{k}$ are modules, then $V_{1} / V_{2} / \ldots / V_{k}$ denotes a module having the 
same composition factors as $V_{1} \oplus V_{2} \oplus \ldots \oplus V_{k}$. If $j$ of the factors are isomorphic to $W$, we write $W^{j}$ for $W / \ldots / W$.

For $\varepsilon= \pm 1, A_{l}^{\varepsilon}(q)$ denotes a quasisimple group $A_{l}(q)$ if $\varepsilon=+1$, and denotes ${ }^{2} A_{l}(q)$ if $\varepsilon=-1$. A similar interpretation holds for $E_{6}^{\varepsilon}(q), G_{2}^{\varepsilon}(q), B_{2}^{\varepsilon}(q), F_{4}^{\varepsilon}(q)$ and $D_{4}^{\varepsilon}(q)$. For $D_{4}^{\varepsilon}(q)$, we also allow $\varepsilon=3$, in which case $D_{4}^{\varepsilon}(q)$ denotes ${ }^{3} D_{4}(q)$.

We fix the following labelling of Dynkin diagrams, where the darkened nodes represent the short roots:

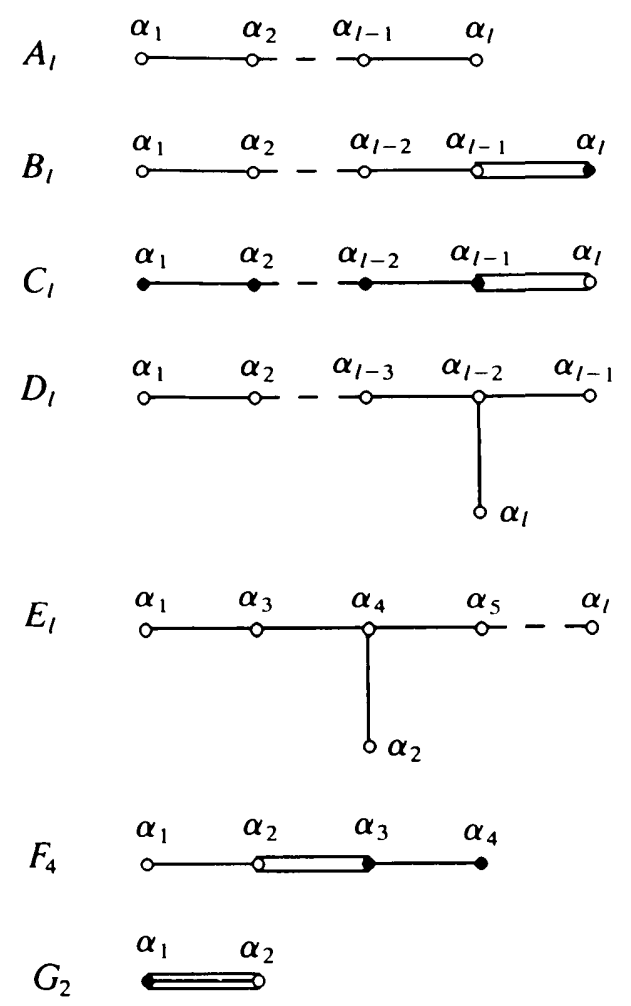

The paper is divided into four further sections. In the first we present some preliminary results needed for the proofs of the theorems. The second, third and fourth sections contain the proofs of Theorems 1,2 and 3 , respectively.

Acknowledgement. We are grateful to Dr Peter Sin for his permission to include his unpublished results, Lemmas 1.7 and 1.9 in $\S 1$. The third author also thanks the Sloane Foundation and the Fonds Nationale Suisse for financial support.

\section{Preliminary lemmas}

In our proofs of Theorems 1 and 2, we shall require detailed information on the cohomology groups $H^{1}(X, V)$, where $V$ is one of several specified modules for the algebraic or finite group $X$ of Lie type. The first nine lemmas contain the information we need. The remaining lemmas of the section are various technical results which we shall require on representations of finite and algebraic groups of Lie type. 
Lemma 1.1. Let $X$ be a simple algebraic group over $k$, and let $V=V_{X}(\lambda)$. If the semidirect product $V X$ contains more than one conjugacy class of closed complements to $V$, then either

(i) $V$ has a rational indecomposable extension by the trivial $k X$-module, or

(ii) $X=C_{n}, p=2$ and $V=V_{X}\left(2^{a} \lambda_{1}\right)\left(\right.$ or $V_{X}\left(2^{a} \lambda_{2}\right)$ if $\left.n=2\right)$, for some $a$.

Proof. See [26, 1.5].

Lemma 1.2. Let $X$ be a finite group, $K$ a field and $M$ a finite-dimensional $K X$-module. Let $W_{1}, \ldots, W_{r}$ be the composition factors of $M$, of which $m$ are trivial, and set $n=\sum \operatorname{dim} H^{1}\left(X, W_{i}\right)$. Assume that $H^{1}(X, K)=0$.

(i) If $n<m$, then $M$ has a trivial $K X$-submodule.

(ii) If $M$ has no trivial submodule and $n=m$, then $H^{1}(X, M)=0$.

Proof. (i) We argue by induction on $r$, the case $r=1$ being trivial. We may suppose that $n>0$, and choose a $K X$-submodule $W$ of $M$, maximal subject to $M / W$ having a non-trivial composition factor. Let $m^{\prime}$ be the number of trivial composition factors of $W$, and $n^{\prime}=\sum \operatorname{dim} H^{1}\left(X, W_{i}\right)$, the sum being over composition factors $W_{i}$ of $W$. If $n^{\prime}<m^{\prime}$ then by induction, $W$ has a trivial $K X$-submodule. Otherwise, $n-n^{\prime}<m-m^{\prime}$, and $M / W$ has a trivial submodule; but this contradicts the choice of $W$.

(ii) We again use induction on $r$. Assume $n>0$, and let $W, m^{\prime}, n^{\prime}$ be as in the proof of (i). Then $n^{\prime} \geqslant m^{\prime}$ by (i). Hence $n-n^{\prime} \leqslant m-m^{\prime}$. By the choice of $W$, $M / W$ has no trivial submodules, so $n-n^{\prime}=m-m^{\prime}$ by (i). By induction then, $H^{1}(X, M / W)=H^{1}(X, W)=0$. Consequently $H^{1}(X, M)=0$.

Lemma 1.3. Let $X$ be a simple algebraic group over $k$, with $(X, p) \neq\left(C_{2}, 2\right)$, $\left(G_{2}, 3\right)$ or $\left(F_{4}, 2\right), V$ a rational $k X$-module such that $H^{1}\left(X, V^{(e)}\right)=0$ for all $e \geqslant 0$. Then $V X$ has only one class of closed complements isomorphic to $X$.

Proof. Let $X^{\prime}$ be a closed complement to $V$ in $V X$ which is isomorphic to $X$. Define $\phi: X^{\prime} \rightarrow X$ to be the composite of the inclusion $X^{\prime} \rightarrow V X$ and the projection $V X \rightarrow X$. Then by $[26,1.2], \phi=F^{\prime} \psi$ where $F^{\prime}$ is a (possibly trivial) field automorphism of $X^{\prime}$ and $\psi$ is a morphism from $X^{\prime}$ to $X$ with $d \psi \neq 0$. If $d \psi: \mathscr{L}\left(X^{\prime}\right) \rightarrow \mathscr{L}(X)$ is not an isomorphism, then $\operatorname{ker}(d \psi)$ and $\operatorname{im}(d \psi)$ are proper ideals; but this is impossible, as we have excluded $(X, p)=\left(C_{2}, 2\right),\left(F_{4}, 2\right)$ and $\left(G_{2}, 3\right)$ (cf. [31,1.2]). Hence, $\psi$ is an isomorphism. Then $F=\psi^{-1} F^{\prime} \psi$ is a field automorphism of $X$, and $\phi=\psi F$.

Write $X^{\prime}=\{x \gamma(x) \mid x \in X\}$, where $\gamma: X \rightarrow V$ is a 1-cocyle, and denote by $\pi: X^{\prime} \rightarrow V$ the composite of the inclusion $X^{\prime} \rightarrow V X$ and the projection $V X \rightarrow V$. Now define $\gamma^{\prime}: X \rightarrow V$ by

$$
\gamma^{\prime}((x \gamma(x)) \psi)=\gamma(x), \quad \text { for } x \in X
$$

Thus $\gamma^{\prime}(y)=\gamma\left(y \psi^{-1} \pi\right)$ for $y \in X$, so $\gamma^{\prime}$ is a rational map. Also $\gamma^{\prime}$ is a 1-cocyle 
for the action of $X$ on the twisted module $V^{F}$ : for if $y_{i}=\left(x_{i} \gamma\left(x_{i}\right)\right) \psi$ for $i=1,2$ $\left(x_{i} \in X\right)$, then

$$
\begin{aligned}
\gamma^{\prime}\left(y_{1} y_{2}\right) & =\gamma\left(x_{1} x_{2}\right) \\
& =\gamma\left(x_{1}\right)^{x_{2}}+\gamma\left(x_{2}\right) \\
& =\gamma^{\prime}\left(y_{1}\right)^{y_{2} F}+\gamma^{\prime}\left(y_{2}\right) .
\end{aligned}
$$

As $H^{1}\left(X, V^{F}\right)=0$ by hypothesis, there exists $v \in V$ such that

$$
\gamma^{\prime}(y)=v-v^{y F}, \text { for all } y \in X .
$$

Since $\gamma^{\prime}(y)=\gamma(y F)$, this implies $\gamma(x)=v-v^{x}$ for all $x \in X$, and so $X^{\prime}=X^{v}$, as required.

Lemma $1.4[12,7.1]$. Suppose that $p>0$, and let $X$ be a simple algebraic group over $k$, defined and split over $\mathbb{F}_{p}$. For $q=p^{a}$ let $X(q)$ be the subgroup of $\mathbb{F}_{q}$-rational points. Let $V$ be a rational $k X$-module. If $X=C_{l}$ with $p=2$, assume that $V$ has no weight of the form $\frac{1}{2} \alpha$ with $\alpha$ a root. Suppose $H^{1}(X(q), V)$ has dimension $n$ for all sufficiently large $q$. Then $H^{1}\left(X, V^{(e)}\right)$ has dimension $n$ for all $e \geqslant 0$.

Lemma 1.5. Let $G$ be a simple algebraic group over $k$, and let $P=Q L$ be $a$ proper parabolic subgroup of $G$ with unipotent radical $Q$ and Levi factor $L$, such that $L^{\prime}$ is simple. Then the $L^{\prime}$-composition factors within $Q$ have the structure of rational $k L^{\prime}$-modules, and the possible high weights $\lambda$ of non-trivial factors are as follows:

(i) $L^{\prime}=A_{n}: \lambda=2 \lambda_{1}, 2 \lambda_{n}, \lambda_{j}$ or $\lambda_{n+1-j}$ for $j=1,2,3$;

(ii) $L^{\prime}=B_{n}$ or $C_{n}: \lambda=\lambda_{1}, \lambda_{2}$ or $\lambda_{3}$, where $\lambda_{2}$ or $\lambda_{3}$ occurs only if $G=F_{4}$ and $n=2$ or $n=3$, respectively;

(iii) $L^{\prime}=D_{n}: \lambda=\lambda_{1}, \lambda_{n-1}$ or $\lambda_{n}$, where $\lambda_{n-1}$ and $\lambda_{n}$ occur only for $G=E_{k}$ and $n \in\{5,6,7\}$

(iv) $L^{\prime}=E_{6}: \lambda=\lambda_{1}$ or $\lambda_{6}$;

(v) $L^{\prime}=E_{7}: \lambda=\lambda_{7}$.

Proof. The module structure is established in [4]. For the possible weights, except $2 \lambda_{1}$ and $2 \lambda_{n}$ when $L^{\prime}=A_{n}$, see $[25,1.4]$. The extra weights $\lambda=2 \lambda_{1}, 2 \lambda_{n}$ for $L^{\prime}=A_{n}$ arise from the short root $A_{n}$ in $G=C_{n+1}$.

LEMMA 1.6. Let $X$ be a simple algebraic group over $k$, and $\lambda$ a dominant weight for $X$ as given in Table 2. Let $V=V_{X}(\lambda)$. Then the semidirect product $V X$ contains only one conjugacy class of closed complements to $V$.

Proof. We apply Lemma 1.1. For all of the given $(X, \lambda)$ apart from $\left(B_{l}, \lambda_{1}\right)$, $\left(C_{l}, 2 \lambda_{1}\right),\left(B_{3}, 2 \lambda_{3}\right),\left(C_{4}, \lambda_{2}\right),\left(D_{4}, \lambda_{2}\right)$ and $\left(F_{4}, \lambda_{4}\right)$, the weight 0 is not a subdominant weight of $\lambda$, so $\operatorname{Hom}_{X}\left(\operatorname{rad}\left(W_{X}(\lambda)\right), k\right)=0$ and so $V$ does not have a rational indecomposable extension by the trivial $k X$-module (see [17, p. 207]). In the remaining cases, we refer to $\left[8\right.$, Table 1] to see that $\operatorname{Hom}_{X}\left(\operatorname{rad}\left(W_{X}(\lambda)\right), k\right)=0$ again. 
TABLE 2

\begin{tabular}{ll}
\hline$X$ & \multicolumn{1}{c}{$\lambda$} \\
\hline$A_{l}, l>1$ & $2 \lambda_{1}, 2 \lambda_{l}, \lambda_{j}$ or $\lambda_{l+1-j}, j=1,2,3$ \\
$B_{l}$ & $2 \lambda_{3}$ for $l=3$ and $p \neq 2, \lambda_{1}$ for $p \neq 2$, or $\lambda_{l}$ \\
$C_{l}$ & $\lambda_{2}$ for $l=4$ and $p \neq 2, \lambda_{1}$ for $p \neq 2,2 \lambda_{1}$ for $p \neq 2$, or $\lambda_{3}$ \\
$D_{l}$ & $\lambda_{2}$ for $l=4$ and $p \neq 2, \lambda_{1}, \lambda_{l-1}$ or $\lambda_{l}$ \\
$F_{4}$ & $\lambda_{4}$ for $p \neq 3$ \\
$E_{6}$ & $\lambda_{1}, \lambda_{6}$ \\
$E_{7}$ & $\lambda_{7}$ \\
\hline
\end{tabular}

The next two lemmas concern $H^{1}\left(X, V_{X}(\lambda)\right)$ for various finite groups $X$ of Lie type. We are grateful to Dr Peter Sin for permission to include his results 1.7 and 1.9.

Lemma 1.7 (P. Sin). (i) If $X=\mathrm{SU}_{3}(3)$, then $H^{1}\left(X, V_{X}\left(2 \lambda_{1}\right)\right)$ has dimension 1. (ii) If $X={ }^{2} F_{4}(8)$, then $H^{1}\left(X, V_{X}\left(\lambda_{4}\right)\right)=0$.

Proof. (i) This is proved in [35].

(ii) Let $X={ }^{2} F_{4}(8)$ and $V=V_{X}\left(\lambda_{4}\right)$. Choose a parabolic subgroup $P=U L$ of $X$ with unipotent radical $U$ and Levi subgroup $L={ }^{2} B_{2}(8) \times \mathbb{F}_{8}^{*}$. It is enough to show that $H^{1}(P, V)=0$.

We first compute the composition factors of $\left.V\right|_{P}$. Write $W=V_{L} \cdot\left(\lambda_{2}\right)$, the usual 4-dimensional module for $L^{\prime}={ }^{2} B_{2}(8)$, and let $[a]$ be the 1-dimensional module for $\mathbb{F}_{8}^{*}$ on which $t \in \mathbb{F}_{8}^{*}$ acts as the scalar $t^{a}$. Calculation shows that the composition factors of $\left.V\right|_{P}$ on which $L^{\prime}$ acts non-trivially are

$$
W \otimes[-1], W \otimes[1], W \otimes[3], W \otimes[-3], W^{(2)} \otimes[0],
$$

while those on which $L^{\prime}$ acts trivially are

$$
\text { [0], [0], [3], [-3], [2], [-2]. }
$$

If $M$ is any irreducible $L^{\prime}$-module, we have an inflation-restriction sequence

$$
0 \rightarrow H^{1}(L, M \otimes[a]) \rightarrow H^{1}(P, M \otimes[a]) \rightarrow H^{1}(U, M \otimes[a])^{L} .
$$

The first term is zero unless $[a]=[0]$, in which case it is 1-dimensional if $\operatorname{dim} M=4$, and 0 otherwise (see [34]). The third term is isomorphic to $\operatorname{Hom}_{\mathrm{F}_{2} L}(\bar{U}, M \otimes[a])$, where $\bar{U}$ is the Frattini quotient of $U$. Using the commutator relations given in [28], we find that as an $\mathbb{F}_{8} L$-module, $\bar{U}$ is a non-split extension of $W^{(2)} \otimes[2]$ by [2] (in fact $\bar{U}$ is only determined up to Galois conjugacy as an $\mathbb{F}_{8} L$-module). From this we compute that $\operatorname{Hom}_{F_{2} L}(\vec{U}, M \otimes[a])$ is isomorphic to $k$ if $M \otimes[a]$ is $\operatorname{Gal}\left(\mathbb{F}_{8}: \mathbb{F}_{2}\right)$-conjugate to $W^{(2)} \otimes[2]$, and is 0 otherwise.

From the inflation-restriction sequence given above, we now see that $H^{1}\left(P, W^{(2)} \otimes[0]\right)$ and $H^{1}(P, W \otimes[-3])$ are both 1-dimensional, and that $H^{1}(P, M \otimes[a])$ vanishes for all other composition factors of $\left.V\right|_{P}$. Lemma 1.2 now applies to give $H^{1}(P, V)=0$, as required. 
TABLE 3

\begin{tabular}{ll}
\hline \multicolumn{1}{c}{$X$} & \multicolumn{1}{c}{$\lambda$} \\
\hline$A_{l}^{\varepsilon}(q)$ & $\lambda_{1}, \lambda_{2}$ \\
& $\lambda_{1}+\lambda_{l}$ for $q \neq 3$ and $p \nmid(l+1)$ \\
& $2 \lambda_{1}$ for $q$ odd, $X \neq{ }^{2} A_{2}(3)$ \\
$B_{l}(q), C_{l}(q), q$ odd & $\lambda_{1}$ for $X \neq B_{2}(3)$ \\
$D_{l}^{\varepsilon}(q)$ & $\lambda_{1}$ \\
$B_{4}(q), q$ odd & $\lambda_{4}$ \\
$C_{4}(q)$ & $\lambda_{2}$ for $p \neq 2, \lambda_{4}$ for $p=2$ \\
$D_{4}^{\varepsilon}(q)$ & $\lambda_{2}$ for $p \neq 2$ and $q \neq 3$ \\
$F_{4}(q)$ & $\lambda_{4}$ for $p \neq 3$ \\
${ }^{2} F_{4}(q)$ & $\lambda_{4}$ \\
$B_{3}(q), q$ odd & $\lambda_{3}$ \\
$C_{3}(q)$ & $\lambda_{2}$ for $p \neq 3, \lambda_{3}$ \\
\hline
\end{tabular}

LemMA 1.8. Let $X$ be a quasisimple group of Lie type over $\mathbb{F}_{q}$, with $q>2$. Let $\lambda$ be a dominant weight and $V=V_{X}(\lambda)$, the corresponding irreducible $k X$-module.

(a) In the cases listed in Table 3, $H^{1}(X, V)=0$.

(b) In the cases listed in Table 4. $\operatorname{dim} H^{1}(X, V)=1$.

(c) When $X=D_{4}^{\varepsilon}(q), \lambda=\lambda_{2}$ and $p=2, \operatorname{dim} H^{1}(X, V)=2$.

TABLE 4

\begin{tabular}{cll}
\hline$X$ & \multicolumn{1}{c}{$\lambda$} & \multicolumn{1}{c}{$p$} \\
\hline$A_{l}^{\varepsilon}(q)$ & $\lambda_{1}+\lambda_{l}$ & $p \mid l+1$ and $q \neq 3$ \\
$C_{l}(q)$ & $\lambda_{1}$ & 2 \\
$C_{4}(q)$ & $\lambda_{2}$ & 2 \\
$C_{3}(q)$ & $\lambda_{2}$ & 3 \\
$B_{2}(3)$ & $\lambda_{1}$ & 3 \\
$F_{4}(q)$ & $\lambda_{4}$ & 3 \\
\hline
\end{tabular}

Proof. This follows from $[18 ; 19 ; 25,1.8]$ and Lemma 1.7 , except for the case where $X=A_{l}^{\varepsilon}(q), \lambda=2 \lambda_{1}$ and either $q>3$ or $q=3, \varepsilon=-1, l>2$. In this case, let $V=V_{X}\left(2 \lambda_{1}\right)$, so that $V$ is the symmetric square of the natural module for $X$, and write $F=\mathbb{F}_{q}$ if $\varepsilon=1, F=\mathbb{F}_{q^{2}}$ if $\varepsilon=-1$. Choose $\zeta \in F$ of order $q-\varepsilon$. We use the argument of $[25,1.8]$ (originally in [1]); thus we define abelian $p^{\prime}$-subgroups $E_{1}$, $E_{2}$ of $X$ such that each $E_{i}$ is fixed-point-free on $V-\{0\},\left[E_{1}, E_{2}\right]=1$ and $X=\left\langle C_{X}\left(E_{i}\right) \mid i=1,2\right\rangle$. It then follows as in $[25,1.8]$ that $H^{1}(X, V)=0$.

If $\zeta^{l} \neq \pm 1$ and $\zeta^{l-1} \neq 1$, take

$$
E_{1}=\left\langle\operatorname{diag}\left(\zeta, \ldots, \zeta, \zeta^{-l}\right)\right\rangle, \quad E_{2}=\left\langle\operatorname{diag}\left(\zeta^{-\ell}, \zeta, \ldots, \zeta\right)\right\rangle
$$

(matrices relative to an orthornormal basis of $V$ if $\varepsilon=-1$ ). If $\zeta^{l}= \pm 1$ and $(q, \varepsilon) \neq(5,+1)$ or $(3,-1)$, take

$$
E_{1}=\left\langle\operatorname{diag}\left(\zeta^{2}, \ldots, \zeta^{2}, \zeta, \zeta\right)\right\rangle, \quad E_{2}=\left\langle\operatorname{diag}\left(\zeta, \zeta^{2}, \ldots, \zeta^{2}, \zeta\right)\right\rangle
$$


If $\zeta^{l-1}=1$ and $(q, \varepsilon) \neq(5,+1)$ or $(3,-1)$, take

$$
E_{1}=\left\langle\operatorname{diag}\left(\zeta^{2}, \ldots, \zeta^{2}, \zeta, \zeta, \zeta, \zeta\right)\right\rangle, \quad E_{2}=\left\langle\operatorname{diag}\left(\zeta, \zeta^{2}, \ldots, \zeta^{2}, \zeta, \zeta, \zeta\right)\right\rangle
$$

Now suppose $(q, \varepsilon)=(5,+1)$ or $(3,-1)$ and $l>2$. If $l$ is even, let

$$
\begin{aligned}
& E_{1}=\langle\operatorname{diag}(\zeta, \ldots, \zeta, \pm 1), \operatorname{diag}(\zeta, \ldots, \zeta, \pm 1, \zeta)\rangle, \\
& E_{2}=\langle\operatorname{diag}( \pm 1, \zeta, \ldots, \zeta), \operatorname{diag}(\zeta, \pm 1, \zeta, \ldots, \zeta)\rangle .
\end{aligned}
$$

If $l$ is odd, then $\zeta^{l-1}=1$ and $l \equiv 1(\bmod 4)$ and we may take

$$
\begin{aligned}
& E_{1}=\langle\operatorname{diag}(\zeta, \ldots, \zeta,-\zeta), \operatorname{diag}(\zeta, \ldots, \zeta,-1,-1)\rangle, \\
& E_{2}=\langle\operatorname{diag}(-\zeta, \zeta, \ldots, \zeta), \operatorname{diag}(-1,-1, \zeta, \ldots, \zeta)\rangle
\end{aligned}
$$

In all cases, $E_{1}$ and $E_{2}$ satisfy the required conditions (use $[29,3.2]$ to see that $\left.X=\left\langle C_{X}\left(E_{1}\right), C_{X}\left(E_{2}\right)\right\rangle\right)$.

It remains only to deal with the case where $X=A_{2}(5)$. Here, let $H$ be a Cartan subgroup of $X$ and $U$ a Sylow 5-subgroup normalized by $H$. Observe that the weights of $H$ on $V$ are all different from the weights of $H$ on $U$. Hence if $U_{\alpha}$ is a root subgroup of $U$, then it is the unique subgroup of $V H U_{\alpha}$ on which $H$ acts with weight $\alpha$. It follows that there is just one class of complements to $V$ in $V H U$, and this implies that $H^{1}(X, V)=0$.

Lemma 1.9 (P. Sin). Let $X=D_{4}^{\varepsilon}(q)$, with $q$ a power of 3 , and let $V=V_{X}\left(\lambda_{2}\right)$. Then $\operatorname{Ext}_{X}^{1}(V, V)=0$.

Proof. Let $B$ be a Borel subgroup of $X$, and let $P=U L$ be a parabolic subgroup containing $B$, with unipotent radical $U$ and Levi subgroup $L$, where $L=\mathrm{SL}_{2}(q)^{3} \times Z_{q-1}$ if $\varepsilon=+1, L=\mathrm{SL}_{2}(q) \times \mathrm{SL}_{2}\left(q^{2}\right) \times Z_{q-1}$ if $\varepsilon=-1$, and $L=$ $\mathrm{SL}_{2}\left(q^{3}\right) \times Z_{q-1}$ if $\varepsilon=3$.

We first establish that $H^{1}(P, V)=0$. To do this, we use the method of the proof of 1.7(ii): we compute $H^{1}(P, M \otimes[a])$ for each composition factor $M \otimes[a]$ of $\left.V\right|_{P}$, using the inflation-restriction sequence

$$
0 \rightarrow H^{1}(L, M \otimes[a]) \rightarrow H^{1}(P, M \otimes[a]) \rightarrow \operatorname{Hom}_{\mathbb{F}_{3} L}(\bar{U}, M \otimes[a]),
$$

where $\bar{U}$ is the Frattini quotient of $U$. When $q=3$, we find that there are two composition factors with $H^{1}(P, M \otimes[a]) \cong k$ and the rest have trivial 1 cohomology. Since $\left.V\right|_{P}$ has three trivial composition factors and $V^{P}$ is 1 dimensional, Lemma 1.2 applies to give $H^{1}(P, V)=0$ in this case. And if $q>3$, all the composition factors have trivial 1-cohomology, giving the same conclusion.

Now suppose we have a short exact sequence of $k X$-modules

$$
0 \rightarrow V \rightarrow E \rightarrow V \rightarrow 0 \text {. }
$$

As $H^{1}(P, V)=0$, the fixed space $E^{U}$ has dimension 2 ; and since $V \cong V^{*}$, we also have $\operatorname{dim}\left(E^{*}\right)^{U}=2$. Therefore, if $E$ (and hence also $E^{*}$ ) is not split, then $E$ is generated as a $k X$-module by a $B$-fixed line, and so is $E^{*}$. Now Corollary 2 of [38] (see also Theorem 15 of [9]) applies to show that $E$ splits after all.

The next two lemmas concern large-rank subgroups of classical groups and representations of simple algebraic groups of degree at most 56. 
LeMma 1.10. Let $G$ be a classical simple algebraic group over $k$, and let $V$ be the natural irreducible module for $G$ over $k$. Suppose that $M$ is a proper, simple, closed, connected subgroup of $G$ such that $M$ is irreducible on $V$ and $\operatorname{rank}(M)>$ $\frac{1}{2} \operatorname{rank}(G)$. Then $M$ and $G$ are as in Table 5; we also give $\lambda$, where $\left.V\right|_{M}=V_{M}(\lambda)$, and $\lambda$ (a dominant weight for $M$ ) is determined up to an automorphism of $M$ as an abstract group.

\section{TABLE 5}

\begin{tabular}{lll}
\hline$G$ & \multicolumn{1}{c}{$M$} & \multicolumn{1}{c}{$\lambda$} \\
\hline$B_{3}$ & $A_{2}, p=3$ & $\lambda_{1}+\lambda_{2}$ \\
$B_{3}$ & $G_{2}$ & $\lambda_{1}$ \\
$C_{3}$ & $G_{2}, p=2$ & $\lambda_{1}$ \\
$D_{4}$ & $B_{3}$ & $\lambda_{3}$ \\
$A_{2 n-1}$ & $C_{n}, D_{n}$ & $\lambda_{1}$ \\
$C_{n}$ & $D_{n}, p=2$ & $\lambda_{1}$ \\
\hline
\end{tabular}

Proof. The hypothesis that $\operatorname{rank}(M)>\frac{1}{2} \operatorname{rank}(G)$ gives

$$
\operatorname{dim} V \leqslant \begin{cases}2(\operatorname{rank}(M)) & \text { if }\left.V\right|_{M} \text { is not self-dual, } \\ 4(\operatorname{rank}(M))-1 & \text { if }\left.V\right|_{M} \text { is self-dual. }\end{cases}
$$

Hence $\lambda$ is given by $[21, \S 2]$ : either $M$ is $C_{n}$ or $D_{n}$ and $\left.V\right|_{M}$ is the natural $2 n$-dimensional module for $M$, or $(M, \lambda)$ is one of $\left(A_{2}, \lambda_{1}+\lambda_{2}\right)$ with $p=3$, $\left(B_{3}, \lambda_{3}\right),\left(C_{3}, \lambda_{3}\right)$ with $p=2,\left(G_{2}, \lambda_{1}\right)$, or $\left(G_{2}, \lambda_{2}\right)$ with $p=3$. All of these give rise to groups in the conclusion. (Note that when $p=2,\left(C_{3}, \lambda_{3}\right)$ gives the same subgroup $M$ of $D_{4}$ as $\left(B_{3}, \lambda_{3}\right)$; so the conclusion gives only one of these cases.)

LEMMA 1.11. Let $X$ be a simple algebraic group over $k$ of rank $l=3$ or 4 , with $(X, p) \neq\left(B_{l}, 2\right)$. Suppose that $V=V_{X}(\lambda)$ is a non-trivial irreducible $k X$-module such that

$$
\operatorname{dim} V \leqslant \begin{cases}56 & \text { if } l=4 \text { and } V \text { is self-dual, } \\ 26 & \text { if } l=3 \text { and } V \text { is self-dual, } \\ 28 & \text { if } l=4 \text { and } V \text { is not self } \text {-dual, } \\ 13 & \text { if } l=3 \text { and } V \text { is not self } \text {-dual. }\end{cases}
$$

Then $X, \lambda$ and $\operatorname{dim} V_{X}(\lambda)$ are as listed in Table 6 , with $\lambda$ given up to an automorphism of $X$ as an abstract group.

Proof. Consider first the case where $\lambda$ is a restricted weight for $X$. Counting conjugates of $\lambda$ under the Weyl group, and of subdominant weights which are known to occur as weights of $V_{X}(\lambda)$ (for example, using $[37,1.30]$ ), we see that either $\lambda$ is as listed in the conclusion, or $(X, \lambda)$ is one of: $\left(A_{4}, \lambda_{2}+\lambda_{3}\right)$, $\left(D_{4}, \lambda_{1}+\lambda_{2}\right),\left(A_{3}, \lambda_{1}+\lambda_{2}\right),\left(B_{3}, 2 \lambda_{3}\right),\left(C_{3}, \lambda_{1}+\lambda_{3}\right.$ or $\left.\lambda_{2}+\lambda_{3}\right)$.

Now refer to [8] to see that $p=3$ and $\operatorname{dim} V=51$ in the first case, while the dimensions of the remaining modules $V_{X}(\lambda)$ are too large.

When $\lambda$ is not restricted and is not a Frobenius twist of a restricted weight, $V$ is 
TABLE 6

\begin{tabular}{ll}
\hline$X$ & \multicolumn{1}{c}{$\left(\lambda, \operatorname{dim} V_{X}(\lambda)\right)$} \\
\hline$A_{4}$ & $\begin{array}{l}\left(\lambda_{1}, 5\right),\left(2 \lambda_{1}(p \neq 2), 15\right),\left(\lambda_{2}, 10\right),\left(\lambda_{1}+\lambda_{4}, 24 \text { for } p \neq 5\right),\left(\lambda_{1}+\lambda_{4}, 23 \text { for } p=5\right), \\
\left(\lambda_{i}+p^{a} \lambda_{j}, 25(i, j \in\{1,4\}, a>0)\right),\left(\lambda_{2}+\lambda_{3}(p=3), 51\right)\end{array}$ \\
$B_{4}$ & $\begin{array}{l}\left(\lambda_{1}, 9\right),\left(\lambda_{2}, 36\right),\left(\lambda_{4}, 16\right),\left(2 \lambda_{1}, 44 \text { for } p \neq 3\right),\left(2 \lambda_{1}, 43 \text { for } p=3\right) \\
C_{4}\end{array}$ \\
& $\begin{array}{l}\left(\lambda_{1}, 8\right),\left(\lambda_{2}, 27 \text { for } p \neq 2\right),\left(\lambda_{2}, 26 \text { for } p=2\right),\left(\lambda_{3}, 48 \text { for } p \neq 3\right),\left(\lambda_{3}, 40 \text { for } p=3\right), \\
\left(\lambda_{4}, 42 \text { for } p \neq 2,3\right),\left(\lambda_{4}, 41 \text { for } p=3\right),\left(\lambda_{4}, 16 \text { for } p=2\right)\end{array}$ \\
$D_{4}$ & $\left(\lambda_{1}, 8\right),\left(\lambda_{2}, 28\right.$ for $\left.p \neq 2\right),\left(\lambda_{2}, 26\right.$ for $\left.p=2\right),\left(2 \lambda_{1}(p \neq 2), 35\right)$, \\
& $\left(\lambda_{1}+\lambda_{3}, 56\right.$ for $\left.p \neq 2\right),\left(\lambda_{1}+\lambda_{3}, 48\right.$ for $\left.p=2\right)$ \\
$F_{4}$ & $\left(\lambda_{4}, 26\right.$ for $\left.p \neq 3\right),\left(\lambda_{4}, 25\right.$ for $\left.p=3\right),\left(\lambda_{1}(p \neq 2), 52\right)$ \\
$A_{3}$ & $\left(\lambda_{1}, 4\right),\left(\lambda_{2}, 6\right),\left(2 \lambda_{1}(p \neq 2), 10\right),\left(\lambda_{1}+\lambda_{3}, 15\right.$ for $\left.p \neq 2\right),\left(\lambda_{1}+\lambda_{3}, 14\right.$ for $\left.p=2\right)$, \\
& $\left(2 \lambda_{2}, 20\right.$ for $\left.p \neq 2,3\right),\left(2 \lambda_{2}, 19\right.$ for $\left.p=3\right)$ \\
$B_{3} \quad\left(\lambda_{1}, 7\right),\left(\lambda_{2}, 21\right),\left(\lambda_{3}, 8\right),\left(2 \lambda_{1}, 26\right.$ for $\left.p=7\right)$ \\
$C_{3} \quad\left(\lambda_{1}, 6\right),\left(\lambda_{2}, 14\right.$ for $\left.p \neq 3\right),\left(\lambda_{2}, 13\right.$ for $\left.p=3\right),\left(\lambda_{3}, 14\right.$ for $\left.p \neq 2\right),\left(\lambda_{3}, 8\right.$ for $\left.p=2\right)$, \\
\end{tabular}

a tensor product of irreducible $\dot{k} X$-modules already found, and it is easy to see that $X=A_{4}$ and $\lambda=\lambda_{1}+p^{i} \lambda_{1}$ or $\lambda_{1}+p^{i} \lambda_{4}$ for some $i \geqslant 1$.

The next two lemmas of this section concern the actions of various elements of $F_{4}$ and $E_{7}$ on modules of dimensions 26 and 56, respectively.

LEMMA 1.12. Let $G$ be a simple algebraic group of type $F_{4}$ or $E_{7}$ over $k$, with $p>0$. Let $V=V(\lambda)$ be an irreducible $k G$-module of high weight $\lambda_{4}$ if $G=F_{4}, \lambda_{7}$ if $G=E_{7}$. Then the dimensions $\operatorname{dim} C_{V}(t)$ for certain conjugacy classes of semisimple elements $t \in G$ are given in Table 7 .

TABLE 7

\begin{tabular}{llll}
\hline \multicolumn{1}{c}{$G$} & \multicolumn{1}{c}{$p$} & $o(t)$ & \multicolumn{1}{c}{$\operatorname{dim} C_{V}(t)$} \\
\hline$F_{4}$ & odd & 2 & $10,14(9,13$ if $p=3)$ \\
$E_{7}$ & 2 & 3 & 8,14 \\
(simply connected) & 2 & 3 & $2,14,20,32$ \\
\hline
\end{tabular}

Proof. Let $G=F_{4}$ with $p$ odd. By [16], there exist two conjugacy classes of involutions in $G$ with centralizers of types $B_{4}$ and $C_{3} A_{1}$. We note that $\left.V\right|_{B_{4}}$ has composition factors $V\left(\lambda_{4}\right) / V\left(\lambda_{1}\right) / V(0)^{c}$, with $c=0$ or 1 as $p=3$ or not, and $\left.V\right|_{C_{3} A_{1}}$ has composition factors $V_{C_{3}}\left(\lambda_{2}\right) / V_{C_{3}}\left(\lambda_{1}\right) \otimes V_{A_{1}}\left(\lambda_{1}\right)$. The assertion then follows.

Now let $G=F_{4}$ with $p=2$. There exist three classes of elements of order 3, 
represented by $t_{1}, t_{2}, t_{3}$ with respective centralizers $B_{3} T_{1}, C_{3} T_{1}$ and $A_{2} \tilde{A}_{2}$ (cf. $[15, \S 34])$. Since $\left.V\right|_{A_{2} \bar{A}_{2}}$ has composition factors

$$
V_{A_{2}}\left(\lambda_{1}\right) \otimes V_{\tilde{A}_{2}}\left(\lambda_{1}\right) / V_{A_{2}}\left(\lambda_{2}\right) \otimes V_{\tilde{A}_{2}}\left(\lambda_{2}\right) / V_{\bar{A}_{2}}\left(\lambda_{1}+\lambda_{2}\right),
$$

$\operatorname{dim} C_{V}\left(t_{3}\right)=8$. There is a $C_{3} A_{1}$ containing the $C_{3} T_{1}$, and $\left.V\right|_{C_{3} A_{1}}$ is given above, so $\operatorname{dim} C_{V}\left(t_{2}\right)=14$. Similarly, $B_{3} T_{1}<B_{3} A_{1}$ with $\left.V\right|_{B_{3} A_{1}}$ having composition factors

$$
V_{B_{3}}\left(\lambda_{3}\right) \otimes V_{A_{1}}\left(\lambda_{1}\right) / V_{A_{1}}\left(\lambda_{1}\right) / V_{B_{3}}\left(\lambda_{1}\right) / V(0)^{2} \text {. }
$$

So $\operatorname{dim} C_{V}\left(t_{1}\right)=8$.

Now let $G=E_{7}$ and set $V=V_{G}\left(\lambda_{7}\right)$. Consider first the case where $p$ is odd. There are three classes of involutions in $G$ : one acting as $-I$ on $V$ and the other two having centralizer $A_{1} D_{6}$. Since $\left.V\right|_{A_{1} D_{6}}=V_{D_{6}}\left(\lambda_{5}\right) / V_{A_{1}}\left(\lambda_{1}\right) \otimes V_{D_{6}}\left(\lambda_{1}\right), C_{V}(t)$ has dimension 24 or 32 in the latter case.

Finally, let $G=E_{7}$ with $p=2$. There are five classes of elements of order 3 in $G$, with centralizers $A_{2} A_{5}, T_{1} E_{6}, T_{1} A_{6}, T_{1} D_{6}$ and $T_{1} A_{1} D_{5}$ (cf. [15]). Refer to $[31,1.8]$ to see that

$$
\begin{aligned}
\left.V\right|_{A_{2} A_{5}} & =V_{A_{2}}\left(\lambda_{1}\right) \otimes V_{A_{5}}\left(\lambda_{1}\right) / V_{A_{2}}\left(\lambda_{2}\right) \otimes V_{A_{5}}\left(\lambda_{5}\right) / V_{A_{5}}\left(\lambda_{3}\right), \quad \text { and } t \in Z\left(A_{2}\right) \cap Z\left(A_{5}\right), \\
\left.V\right|_{E_{6}} & =V\left(\lambda_{1}\right) / V\left(\lambda_{6}\right) / V(0)^{2}, \quad \text { and } t \in Z\left(E_{6}\right), \\
\left.V\right|_{A_{7}} & =V\left(\lambda_{2}\right) / V\left(\lambda_{6}\right), \quad \text { and } t \in T_{1}=Z\left(T_{1} A_{6}\right)<A_{7}, \\
\left.V\right|_{A_{1} D_{6}} & =V_{A_{1}}\left(\lambda_{1}\right) \otimes V_{D_{6}}\left(\lambda_{1}\right) / V_{D_{6}}\left(\lambda_{5}\right), \quad \text { and } t \in T_{1}<A_{1} \text { or } t \in D_{6} \text { with } C_{D_{6}}(t)=T_{1} D_{5} .
\end{aligned}
$$

So $C_{V}(t)$ has dimension $20,2,14,32,20$ in the respective cases.

The next lemma concerns the unipotent conjugacy classes of a simple algebraic group of type $E_{7}$. In the statement we use the parameterization of unipotent classes given by [5] for $p$ odd and by [3] for $p=2$ (note that by [27], the Bala-Carter parametrization holds also when $p=3$ ). The lemma lists the Jordan block structures of elements of order $p$ acting on the 56-dimensional module $V_{E_{7}}\left(\lambda_{7}\right)$; this information is taken directly from [20, Table 7].

LEMMA 1.13. Let $G$ be a simple algebraic group of type $E_{7}$ over $k$, and assume that $2 \leqslant p \leqslant 7$. Let $V$ be the irreducible $k G$-module $V_{G}\left(\lambda_{7}\right)$. Then the Jordan canonical forms of all of the conjugacy classes of elements of order $p$ acting on $V$ are listed in Table 8, where $a^{b}$ abbreviates the fact that there are $b a \times a$ blocks.

Lemma 1.14. Let $A=\mathrm{SL}_{2}(k)$, and suppose $p>0$. Let $\phi: A \rightarrow \operatorname{SL}(V)$ be a finite-dimensional rational representation such that all $A$-composition factors of $V$ have restricted high weights. Let $u$ be an element of order $p$ in $A$. Then there exists a closed connected subgroup $U$ of $A$, with $u \in U$, such that any $u$-invariant subspace of $V$ is in fact $U$-invariant.

Proof. By the assumption on the high weights, $V$ is a completely reducible $k A$-module. We may take $u$ to be $\left(\begin{array}{ll}1 & 1 \\ 0 & 1\end{array}\right)$. Let $\left\{V_{0}, \ldots, V_{p-1}\right\}$ be the full set of restricted irreducible modules for $\mathrm{SL}_{2}(k)$, where $V_{i}$ is the space of $i$ th-degree homogeneous polynomials in two indeterminates. Then $V=\bigoplus W_{j}$, with each $W_{j}$ 
TABLE 8

\begin{tabular}{|c|c|c|c|c|c|}
\hline $\begin{array}{l}\text { Conjugacy } \\
\text { class }\end{array}$ & $p$ & $\begin{array}{c}\text { Jordan } \\
\text { decomposition }\end{array}$ & $\begin{array}{l}\text { Conjugacy } \\
\text { class }\end{array}$ & $p$ & $\begin{array}{c}\text { Jordan } \\
\text { decomposition }\end{array}$ \\
\hline$A_{1}$ & $2,3,5,7$ & $1^{32}, 2^{12}$ & $D_{4}\left(a_{1}\right)$ & 5,7 & $1^{8}, 3^{6}, 5^{6}$ \\
\hline \multirow[t]{2}{*}{$2 A_{1}$} & 2 & $1^{16}, 2^{20}$ & $D_{4}$ & 7 & $1^{14}, 7^{6}$ \\
\hline & $3,5,7$ & $1^{18}, 2^{16}, 3^{2}$ & $D_{4}\left(a_{1}\right)+A_{1}$ & 5 & $2^{5}, 3^{4}, 4^{1}, 5^{6}$ \\
\hline$\left(3 A_{1}\right)^{\prime}$ & $\begin{array}{l}2 \\
3,5,7\end{array}$ & $\begin{array}{l}1^{8}, 2^{24} \\
1^{14}, 2^{12}, 3^{6}\end{array}$ & & 7 & $\begin{array}{c}2^{5}, 3^{4}, 4^{2} \\
5^{4}, 6^{1}\end{array}$ \\
\hline$\left(3 A_{1}\right)^{\prime \prime}$ & $\begin{array}{l}2 \\
3 \\
5,7\end{array}$ & $\begin{array}{l}2^{28} \\
2^{25}, 3^{2} \\
2^{26}, 4^{1}\end{array}$ & $A_{3}+A_{2}$ & 5 & $\begin{array}{c}1^{2}, 2^{2}, 3^{4} \\
4^{2}, 5^{6} \\
1^{2}, 2^{2}, 3^{4}, 4^{4},\end{array}$ \\
\hline$A_{2}$ & $3,5,7$ & $1^{20}, 3^{12}$ & & & $5^{2}, 6^{2}$ \\
\hline \multirow[t]{3}{*}{$4 A_{1}$} & 2 & $2^{28}$ & $A_{4}$ & 5 & $1^{6}, 5^{10}$ \\
\hline & 3 & $1^{6}, 2^{13}, 3^{8}$ & & 7 & $1^{6}, 3^{2}, 5^{6}, 7^{2}$ \\
\hline & 5,7 & $1^{6}, 2^{14}, 3^{6}, 4^{1}$ & $A_{3}+A_{2}+A_{1}$ & 5 & $2^{5}, 4^{4}, 5^{6}$ \\
\hline \multirow[t]{2}{*}{$A_{2}+A_{1}$} & 3 & $1^{8}, 2^{6}, 3^{12}$ & & 7 & $2^{5}, 4^{7}, 6^{3}$ \\
\hline & 5,7 & $1^{8}, 2^{8}, 3^{8}, 4^{2}$ & $\left(A_{5}\right)^{\prime}$ & 7 & $1^{4}, 6^{4}, 7^{4}$ \\
\hline \multirow[t]{2}{*}{$A_{2}+2 A_{1}$} & 3 & $1^{6}, 2^{4}, 3^{14}$ & $\left(A_{5}\right)^{\prime \prime}$ & 7 & $6^{7}, 7^{2}$ \\
\hline & 5,7 & $1^{6}, 2^{8}, 3^{6}, 4^{4}$ & $D_{4}+A_{1}$ & 7 & $1^{4}, 2^{5}, 7^{6}$ \\
\hline$A_{3}$ & 5,7 & $1^{14}, 5^{2}, 4^{8}$ & $A_{4}+A_{1}$ & 5 & $1^{2}, 2^{2}, 5^{10}$ \\
\hline \multirow[t]{2}{*}{$2 A_{2}$} & 3 & $1^{2}, 3^{18}$ & & 7 & $1^{2}, 2^{2}, 3^{2}, 4^{2}$ \\
\hline & 5,7 & $1^{4}, 5^{2}, 3^{14}$ & & & $5^{2}, 6^{2}, 7^{2}$ \\
\hline \multirow[t]{2}{*}{$A_{2}+3 A_{1}$} & 3 & $2^{7}, 3^{14}$ & $D_{5}\left(a_{1}\right)$ & 7 & $1^{4}, 2^{2}, 3^{2}, 7^{6}$ \\
\hline & 5,7 & $2^{14}, 4^{7}$ & $A_{4}+A_{2}$ & 5 & $3^{2}, 5^{10}$ \\
\hline \multirow[t]{2}{*}{$\left(A_{3}+A_{1}\right)^{\prime}$} & 5,7 & $1^{6}, 2^{4}, 3^{2}$ & & 7 & $3^{6}, 5^{2}, 7^{4}$ \\
\hline & & $4^{4}, 5^{4}$ & $A_{5}+A_{1}$ & 7 & $5^{2}, 6^{3}, 7^{4}$ \\
\hline \multirow[t]{2}{*}{$\left(A_{3}+A_{1}\right)^{\prime \prime}$} & 5 & $2^{7}, 4^{8}, 5^{2}$ & $A_{6}$ & 7 & $7^{8}$ \\
\hline & 7 & $2^{7}, 4^{9}, 6^{1}$ & $D_{5}\left(a_{1}\right)+A_{1}$ & 7 & $2^{5}, 4^{1}, 7^{6}$ \\
\hline \multirow[t]{3}{*}{$A_{1}+2 A_{2}$} & 3 & $1^{2}, 3^{18}$ & $D_{6}\left(a_{2}\right)$ & 7 & $4^{1}, 5^{2}, 7^{6}$ \\
\hline & 5,7 & $1^{4}, 2^{4}, 3^{6}$ & $E_{6}\left(a_{3}\right)$ & 7 & $1^{4}, 5^{2}, 7^{6}$ \\
\hline & & $4^{4}, 5^{2}$ & $E_{7}\left(a_{5}\right)$ & 7 & $4^{2}, 6^{1}, 7^{6}$ \\
\hline \multirow[t]{2}{*}{$A_{3}+2 A_{1}$} & 5 & $\begin{array}{c}1^{2}, 2^{3}, 3^{4} \\
4^{4}, 5^{4}\end{array}$ & & & \\
\hline & 7 & $\begin{array}{l}1^{2}, 2^{3}, 3^{4} \\
4^{5}, 5^{2}, 6^{1}\end{array}$ & & & \\
\hline
\end{tabular}

isomorphic to some $V_{i}$. Let $\phi_{j}: A \rightarrow \operatorname{SL}\left(W_{j}\right)$ so that $\phi$ is the direct sum of the $\phi_{j}$. Write

$$
e_{j}=\left(\varphi_{j}(u)-1\right)-\frac{1}{2}\left(\phi_{j}(u)-1\right)^{2}+\ldots-\frac{1}{p-1}\left(\phi_{j}(u)-1\right)^{p-1} \in \mathrm{sl}\left(W_{j}\right)
$$

and set $e=\bigoplus e_{j} \in \operatorname{sl}(V)$. Since $\left(\phi_{j}(u)-1\right)^{p}=0$ for all $j$, and $e^{p}=0$, we have that

$$
\exp (e)=\sum_{k \geq 0} \frac{1}{k !} e^{k}=\phi(u)
$$


We claim that for $t \in k$,

$$
\exp (t e)=\sum_{k \geqslant 0} \frac{1}{k !}(t e)^{k} \in \phi(A)
$$

in fact,

$$
\exp (t e)=\phi\left(\left[\begin{array}{ll}
1 & t \\
0 & 1
\end{array}\right]\right)
$$

For this, it will suffice to show that

$$
\exp \left(t e_{j}\right)=\phi_{j}\left(\left[\begin{array}{ll}
1 & t \\
0 & 1
\end{array}\right]\right)
$$

for each $j$. Suppose $W_{j}=V_{k}$. Set $M_{k}$ to be the $(k+1) \times(k+1)$ matrix whose $(n, m)$ entry is 0 if $m \neq n+1$ and is $n$ otherwise. Also set $h(c)=\left(\begin{array}{cc}c & 0 \\ 0 & c^{-1}\end{array}\right) \in A$.

Then with respect to the ordered basis $x^{k}, x^{k-1} y, \ldots, y^{k}$ of $V_{k}$, we have $\exp \left(M_{k}\right)=\phi_{j}(u)$ and also $c^{2} M_{k}=\phi_{j}(h(c)) M_{k} \phi_{j}\left(h\left(c^{-1}\right)\right)$ for all $c \in k$. We deduce that there exists an ordered basis of $W_{j}$ with respect to which $e_{j}=M_{k}$ and also $c^{2} e_{j}=\phi_{j}(h(c)) e_{j} \phi_{j}\left(h\left(c^{-1}\right)\right)$ for all $c \in k$. Therefore

$$
\begin{aligned}
\exp \left(c^{2} e_{j}\right) & =\phi_{j}(h(c)) \exp \left(e_{j}\right) \phi_{j}\left(h\left(c^{-1}\right)\right) \\
& =\phi_{j}(h(c)) \phi_{j}(u) \phi_{j}\left(h\left(c^{-1}\right)\right) \\
& =\phi_{j}\left[\begin{array}{ll}
1 & c^{2} \\
0 & 1
\end{array}\right] \quad \text { for all } c \in k
\end{aligned}
$$

as claimed.

Clearly $\{\exp (t e) \mid t \in k\}$ is a closed connected 1-dimensional subgroup of $\phi(A)$ containing $\phi(u)$. Moreover, any $u$-invariant subspace of $V$ is invariant under the action of $e$ and therefore invariant under the action of $\exp (t e)$.

Remark. This lemma is related to a result of Serre [33,4.1]. Serre's result implies that there is a subgroup $U$ of $\mathrm{GL}(V)$ with the desired property, whereas we produce a subgroup $U$ also lying in our given $\mathrm{SL}_{2}$.

\section{Proof of Theorem 1}

As in the hypotheses of Theorem 1 , let $G$ be a simple algebraic group over the algebraically closed field $k$ of characteristic $p$, and let $X$ be a simple closed connected subgroup of $G$ with $\operatorname{rank}(X)>\frac{1}{2} \operatorname{rank}(G)$. Write $l=\operatorname{rank}(X), n=$ $\operatorname{rank}(G)$, so that $l>\frac{1}{2} n$.

LEMMA 2.1. If Theorem 1 holds for $(G, p)=\left(C_{n}, 2\right)$ then it holds for $(G, p)=$ $\left(B_{n}, 2\right)$.

Proof. As in [36, Theorem 28], let $\Sigma$ be the $B_{n}$ root system and $\Sigma^{*}$ the dual root system (of type $C_{n}$ ), and let $\alpha \rightarrow \alpha^{*}$ be the map from $\Sigma$ to $\Sigma^{*}$ sending $\alpha$ to 
its coroot $\alpha^{*}$. Then by [36, Theorem 28], when $p=2$ there is a morphism of algebraic groups $\theta: B_{n} \rightarrow C_{n}$ such that

$$
\theta\left(x_{\alpha}(t)\right)= \begin{cases}x_{\alpha^{*}}(t) & \text { if } \alpha \text { is long, } \\ x_{\alpha^{*}}\left(t^{2}\right) & \text { if } \alpha \text { is short. }\end{cases}
$$

Moreover, $\theta$ is an isomorphism of abstract groups.

Now suppose Theorem 1 holds when $G=C_{n}$ and $p=2$. If $X$ is a simple, closed subgroup of $B_{n}$ of rank $l>\frac{1}{2} n$, then $\theta(X) \leqslant C_{n}$, so $\theta(X)$ is as in the conclusion of Theorem 1. Therefore either $\theta(X)$ is $Y$ or $Y_{\tau}$ for some subsystem subgroup $Y$ of $C_{n}$, or $n=3$ and $\theta(X)=G_{2}$. In the latter case, $X=G_{2}$, and since there exists a unique conjugacy class of subgroups of type $G_{2}$ in $C_{3}$, the same is true in $B_{3}$, as required. Now suppose $\theta(X)=Y$ or $Y_{\tau}$; so $X=\theta^{-1}(Y)$ or $\theta^{-1}\left(Y_{\tau}\right)$. As $Y$ is generated by root groups corresponding to roots in some subsystem of $\Sigma^{*}, \theta^{-1}(Y)$ is a subsystem subgroup of $B_{n}$. Therefore if $\theta(X)=Y$, then $X$ is as in (I) of Theorem 1. Finally, suppose $X=\theta^{-1}\left(Y_{\tau}\right)$. Then $Y=A_{r}$ or $D_{r}$ is a short root subsystem subgroup of $C_{n}$. If $Y=A_{r}$ then $Y_{\tau}$ has rank at most $\frac{1}{2}(r+1)$, whence $l \leqslant \frac{1}{2} n$, a contradiction. Hence $Y=D_{r}$. Then $\theta^{-1}(Y)$ is a long root subsystem subgroup $D_{r}$ of $B_{n}$, and $X>\theta^{-1}(Y)$ is of type $B_{r-1}=B_{l}$. Let $W$ be the usual $2 r$-dimensional module for $\theta^{-1}(Y)=D_{r}$. If $l \geqslant 4$, then the only non-trivial irreducible $B_{l}$-module of dimension at most $2 r$ is the usual $B_{l}$-module, and hence $X$ fixes a 1-space of $W$; it follows that $X$ is the stabilizer of a non-singular 1-space of $W$, and hence $X=\left(\theta^{-1}(Y)\right)_{\tau^{\prime}}$ for some graph automorphism $\tau^{\prime}$, as in (II) of Theorem 1. If $l=3$, there are three classes of subgroups $B_{3}$ in $D_{4}$, permuted by triality, and the same conclusion follows. Finally, if $l=2$ then subgroups $B_{2}$ in $D_{3}$ correspond to subgroups $C_{2}$ in $A_{3}$, of which there is just one conjugacy class, and the result again follows.

In view of Lemma 2.1 , we assume that $p \neq 2$ when $G=B_{n}$.

We now embark upon the proof of Theorem 1 by induction on $\operatorname{dim} G$. The result is trivial when $G=A_{1}$.

Lemma 2.2. Theorem 1 holds if $X \leqslant H<G$, where $H$ is reductive of maximal rank.

Proof. Let $H^{\prime}=H_{1} \circ \ldots \circ H_{t}$, a commuting product of simple groups $H_{i}$. Since $l>\frac{1}{2} n, X$ lies in one of the factors $H_{i}$. Hence by induction there is a subsystem subgroup $Y$ of $H_{i}$ containing $X$, such that $X$ and $Y$ are as in the conclusion of Theorem 1. Then $Y$ is also a subsystem subgroup of $G$, so the result holds.

In the light of Lemma 2.2, we assume that

$X$ lies in no proper reductive maximal rank subgroup of $G$.

Lemma 2.3. Theorem 1 holds if $X$ is contained in no proper parabolic subgroup of $G$.

Proof. Pick a maximal proper closed connected subgroup $M$ of $G$ containing $X$. By hypothesis, $M$ is not parabolic, so by [7] it is reductive. Moreover $X \leqslant M^{\prime}$, so $\operatorname{rank}\left(M^{\prime}\right)>\frac{1}{2} n$. By $(\dagger), M$ is not of maximal rank. 
Suppose first that $G$ is of exceptional type. Since $M$ is not of maximal rank, [31, Theorem 1] applies to show that $(M, G)$ is $\left(F_{4}, E_{6}\right),\left(C_{4}, E_{6}\right)$ with $p \neq 2$ or $\left(A_{1} F_{4}, E_{7}\right)$. In the last case, $X$ lies in the factor $F_{4}$, which in turn lies in a subsystem subgroup $E_{6}$ of $G$, contrary to $(\dagger)$. By induction, we know the embedding of $X$ in $M$ : since $l>\frac{1}{2} n, l$ must be 4 and $X=B_{4}, C_{4}, D_{4}$ or $F_{4}$. If $X=B_{4}$, then $X=Y_{\tau}$, where $Y$ is a subsystem subgroup $D_{5}$. If $X=F_{4}$ or if $X=C_{4}$ with $p \neq 2$, then $X=Y_{\tau}$ with $Y$ a subsystem subgroup $E_{6}$. If $X=D_{4}$ and $X$ is a long root subsystem subgroup of $M=F_{4}$, then $X$ is a subsystem subgroup of $G$. In the remaining cases, $X=D_{4}$ or $C_{4}$, with $p=2$, and $X$ is a short root subsystem subgroup of $F_{4}$, as in the conclusion (III) of Theorem 1 .

Now suppose $G$ is a classical group, with natural module $V$. If $M$ is reducible on $V$, then as $M$ is not of maximal rank, we have $M=B_{r} \times B_{n-r-1}<G=D_{n}$, for some $r$ with $0 \leqslant r<\frac{1}{2} n$. Since $l>\frac{1}{2} n$, this forces $X \leqslant B_{n-r-1}$. Then by induction, $X=D_{l}$ or $B_{l}$ and satisfies (I) or (II) of Theorem 1 .

Assume finally that $M$ is irreducible on $V$. If $M$ is not simple, then by [30, Theorem 3], $V=V_{1} \otimes V_{2}$ and $M$ is a central product of classical groups on $V_{1}$ and $V_{2}$; but then $X$ lies in one of the factors, so has rank at most $\frac{1}{2} n$. Consequently $M$ is simple. Now Lemma 1.10 determines $M, G$ and the representation of $M$ on $V$. As $l>\frac{1}{2} n$, we conclude that either $X=M$ (satisfying the conclusion of Theorem $1)$, or $(X, M, G)$ is $\left(D_{n}, C_{n}, A_{2 n-1}\right)$ for $p=2$ or $\left(A_{2}, G_{2}, B_{3}\right.$ or $\left.C_{3}\right)$ or $\left(A_{3}, B_{3}, D_{4}\right)$. In the first case, (III) of Theorem 1 holds. In the second case, either $X$ is a long root subsystem subgroup (of type $A_{2}$ ) or $p=3$ and $X$ is a short root subsystem subgroup of $B_{3}$, which is irreducible on $V$; so (I) or (III) of Theorem 1 holds. In the third case, (I) holds. This completes the proof of Lemma 2.3.

We now assume that $X$ lies in a proper parabolic subgroup of $G$, and take $P=Q L$ to be a minimal such parabolic subgroup, where $Q$ is the unipotent radical of $P$ and $L$ is a Levi factor. Choose a closed subgroup $\tilde{X}$ of $L^{\prime}$ such that $Q X=Q \tilde{X}$. Since $\operatorname{rank}(\tilde{X})>\frac{1}{2} n$ and $P$ is minimal, $L^{\prime}$ is simple. By induction, the embedding of $\tilde{X}$ in $L^{\prime}$ is given by the conclusion of Theorem 1 . Thus $\tilde{X}$ is contained in a simple subsystem subgroup $Y$ of $L^{\prime}$, and by the minimality of $P, Y$ lies in no proper Levi subsystem subgroup of $L^{\prime}$. A quick inspection of subsystems shows that either $Y=L^{\prime}$ or $\left(Y, L^{\prime}\right)$ is one of $\left(D_{r}, B_{r}\right),\left(D_{r}, C_{r}\right)(p=2)$ and $\left(A_{7}, E_{7}\right)$. The embedding of $\widetilde{X}$ in $Y$ satisfies (I), (II) or (III) of Theorem 1 (note that (IV) is not possible since $\operatorname{rank}(X)>\frac{1}{2} \operatorname{rank}(G)$ ). We deduce that either $\tilde{X}=L^{\prime}$ or $\left(\tilde{X}, L^{\prime}\right)$ is one of $\left(B_{l}, D_{l+1}\right),\left(D_{l}, B_{l}\right),\left(F_{4}, E_{6}\right),\left(C_{4}, E_{6}\right),\left(A_{7}, E_{7}\right)$, $\left(D_{l}, C_{l}\right)$, or $\left(D_{4}, E_{6}\right)$ (the last two possibilities occurring only for $p=2$ ).

Now let

$$
1=Q_{0}<Q_{1}<\ldots<Q_{m}=Q
$$

be an $L^{\prime}$-invariant series, with each factor $V_{i}=Q_{i} / Q_{i-1}$ an irreducible high weight module for $L^{\prime}$, with possible high weights given by Lemma 1.5. By $(\dagger), X$ is not conjugate to $\tilde{X}$, so the semidirect product $Q \tilde{X}$ has more than one class of closed complements to $Q$, and hence the same is true of $V_{i} \tilde{X}$ for some $i$. The restrictions $\left.V_{i}\right|_{\tilde{X}}$ are easily worked out. From Lemma 1.6 we deduce that $\left(\tilde{X}, L^{\prime}, G, p\right)$ is as in Table 9 . Note that the possibility $L^{\prime}=B_{l}$ with $l>3$ is not included because 
$G \neq B_{n}$ when $p=2$. Observe also that $X$ and $\tilde{X}$ are of the same type, except possibly when they have types $B_{l}, C_{l}$.

TABLE 9

\begin{tabular}{|c|c|c|c|c|}
\hline & $\tilde{X}$ & $L^{\prime}$ & $G$ & $p$ \\
\hline (i) & $C_{1}$ & $C_{l}$ & $C_{n}$ & 2 \\
\hline (ii) & $B_{l}$ & $D_{l+1}$ & $D_{n}, E_{7}$ or $E_{8}$ & 2 \\
\hline (iii) & $B_{3}$ & $B_{3}$ & $F_{4}$ & 2 \\
\hline (iv) & $C_{3}$ & $C_{3}$ & $F_{4}$ & 2 \\
\hline (v) & $B_{4}$ or $C_{4}$ & $E_{6}$ & $E_{7}$ & 2 \\
\hline (vi) & $D_{4}$ & $E_{6}$ & $E_{7}$ & 2 \\
\hline (vii) & $F_{4}$ & $E_{6}$ & $E_{7}$ & 3 \\
\hline
\end{tabular}

LEMMA 2.4. The conclusion of Theorem 1 holds in cases (iii) and (iv) of Table 9.

Proof. First consider (iii). Here $Q$ has an $L^{\prime}$-invariant normal subgroup $R$ of dimension 7 such that $Q / R=V_{L^{\prime}}\left(\lambda_{3}\right)$. It follows, by Lemma 1.6, that $X$ is conjugate to a subgroup of $R L^{\prime}$. But $R L^{\prime}$ lies in a subgroup $B_{4}$ of $F_{4}$, contradicting $(\dagger)$.

Now consider (iv). There is a morphism $\theta: G \rightarrow G$ of the type given in the proof of Lemma 2.1, and $\left(\theta(X), \theta\left(L^{\prime}\right), \theta(G)\right)=\left(B_{3}\right.$ or $\left.C_{3}, B_{3}, G\right)$. Hence by the previous paragraph, $\theta(X)$ lies in a $B_{4}$ subgroup of $G$, and so $X$ lies in a $C_{4}$ subgroup, again contradicting $(\dagger)$.

Lemma 2.5. In cases (ii) and (vii) of Table 9, the conclusion of Theorem 1 holds.

Proof. Consider first case (ii). By Lemmas 1.5 and 1.6, $V_{i} \approx V_{L^{\prime}}\left(\lambda_{1}\right)$, of dimension $2 l+2$. Then $\left.V_{i}\right|_{\bar{X}}$ is indecomposable, with composition factors $V_{\bar{X}}(0) / V_{\bar{X}}\left(2^{a} \lambda_{1}\right) / V_{\bar{X}}(0)$ for some $a \geqslant 0$.

For $q$ a power of 2 , let $\tilde{X}(q) \simeq B_{l}(q) \simeq C_{l}(q)$ be the subgroup of $\mathbb{F}_{q}$-rational points of $\tilde{X}$. By $[\mathbf{1 8}], H^{1}\left(\tilde{X}(q), V_{\tilde{X}}\left(2^{a} \lambda_{1}\right)\right)$ has dimension 1 for all $q$. Hence by Lemma $1.2, H^{1}\left(\tilde{X}(q), V_{i}\right)=0$ for all $q$. Then Lemma 1.4 implies that $H^{1}\left(\tilde{X}, V_{i}^{(e)}\right)=0$ for all $e \geqslant 0$. But now Lemma 1.3 tells us that $V_{i} \tilde{X}$ has only one class of closed complements to $V_{i}$, contrary to our choice of $V_{i}$.

Case (vii) yields to the same argument, since here $Q \approx V_{L^{\prime}}\left(\lambda_{1}\right)$ or $V_{L^{\prime}}\left(\lambda_{6}\right)$ and is indecomposable for $\bar{X}=F_{4}$ with factors $V_{\bar{X}}(0) / V_{\bar{X}}\left(3^{a} \lambda_{1}\right) / V_{\bar{X}}(0)$ (see $[\mathbf{2 5}, 1.5]$ ).

\section{LEMMA 2.6. Theorem 1 holds in case (i) of Table 9.}

Proof. Here $\left(X, L^{\prime}, G, p\right)=\left(B_{l}\right.$ or $\left.C_{l}, C_{l}, C_{n}, 2\right)$. We claim that $X$ contains short root subgroups of $G$. First consider the case where $l>2$. For $j$ with $V_{j}$ a non-trivial $L^{\prime}$-module, we have $V_{j}=V_{L} \cdot\left(\lambda_{1}\right)$. Pick a Levi subgroup $A=A_{l-1}$ of $X$. Then $A$ lies in $V_{j} A^{\prime}$ where $A^{\prime} \simeq A_{l-1}$ is a Levi subgroup of $L^{\prime}$. Then $\left.V_{j}\right|_{A^{\prime}}$ has composition factors $V_{A^{\prime}}\left(\lambda_{1}\right)$ and $V_{A^{\prime}}\left(\lambda_{l-1}\right)$. Since $l-1 \geqslant 2$, Lemma 1.6 implies 
that $V_{j} A^{\prime}$ has only one class of closed complements to $V_{j}$ and hence $A$ is conjugate to $A^{\prime}$. Then $A$, and hence $X$, contains short root subgroups of $G$.

Now consider the case where $l=2$, so $\left(X, L^{\prime}, G, p\right)=\left(C_{2}, C_{2}, C_{3}, 2\right)$. We adopt the following notation. Let $\left\{\alpha_{1}, \alpha_{2}\right\}$ be a base for the root system of $X$, with $\alpha_{2}$ long, and let $\left\{\beta_{1}, \beta_{2}, \beta_{3}\right\}$ be a base of the root system of $G$, with $\beta_{3}$ long. Let $h_{\beta_{1}}(c)$, for $c \in k^{*}$, denote an element of $T(G)$ corresponding to the root $\beta_{i}$ $(i=1,2,3)$. Similarly for $T(X)$ a maximal torus of $X$ and $h_{\alpha_{t}}(c)$. So we may take $L^{\prime}=\left\langle U_{ \pm \beta_{2}}(t), U_{ \pm \beta_{3}}(t) \mid t \in k\right\rangle$ and

$$
Q=\left\langle U_{\beta_{1}}(t), U_{\beta_{1}+\beta_{2}}(t), U_{\beta_{1}+\beta_{2}+\beta_{3}}(t), U_{\beta_{1}+2 \beta_{2}+\beta_{3}}(t), U_{2 \beta_{1}+2 \beta_{2}+\beta_{3}}(t) \mid t \in k\right\rangle .
$$

Now since $X<L^{\prime} Q$, we may assume that one of the following holds (for $q=2^{a}$ for some $a \geqslant 0$ ):

(i) $h_{\alpha_{1}}(c)=h_{\beta_{2}}\left(c^{q}\right), h_{\alpha_{2}}(c)=h_{\beta_{3}}\left(c^{q}\right), U_{\alpha_{1}}(t) \in U_{\beta_{2}}\left(t^{q}\right) Q$ and $U_{\alpha_{2}}(t) \in U_{\beta_{3}}\left(t^{q}\right) Q$, for $c \in k^{*}, t \in k$

(ii) $h_{\alpha_{1}}(c)=h_{\beta_{3}}\left(c^{2 q}\right), h_{\alpha_{2}}(c)=h_{\beta_{2}}\left(c^{q}\right), U_{\alpha_{1}}(t) \in U_{\beta_{3}}\left(t^{2 q}\right) Q$ and $U_{\alpha_{2}}(t) \in U_{\beta_{2}}\left(t^{q}\right) Q$, for $c \in k^{*}, t \in k$.

In case (i), write

$$
\begin{aligned}
U_{\alpha_{1}}(t)= & U_{\beta_{2}}\left(t^{q}\right) U_{\beta_{1}}\left(f_{1}(t)\right) U_{\beta_{1}+\beta_{2}}\left(f_{2}(t)\right) U_{\beta_{1}+\beta_{2}+\beta_{3}}\left(f_{3}(t)\right) \\
& \times U_{\beta_{1}+2 \beta_{2}+\beta_{3}}\left(f_{4}(t)\right) U_{2 \beta_{1}+2 \beta_{2}+\beta_{3}}\left(f_{5}(t)\right)
\end{aligned}
$$

for some polynomials $f_{i}(t) \in k[t]$. Conjugating $U_{\alpha_{1}}(t)$ by $h_{\alpha_{1}}(c)$, we see that $c^{q} f_{i}(t)=f_{i}\left(c^{2} t\right)$, for $i=1,3, c^{-q} f_{j}(t)=f_{j}\left(c^{2} t\right)$, for $j=2,4$, and $f_{5}(t)=f_{5}\left(c^{2} t\right)$ (all of these equations holding for all $c \in k^{*}$ and $\left.t \in k\right)$. Letting $t=1$, we obtain $f_{5}=0$ and $f_{i}(t)=a_{i} i^{\frac{1}{2}}$ for some $a_{i} \in k$. Now conjugate by $h_{\alpha_{2}}(c)$ to see that in fact $a_{i}=0$ for all $i$. Thus $U_{\alpha_{1}}(t)=U_{\beta_{2}}\left(t^{q}\right)$ for all $t \in k$. In case (ii), argue similarly with $U_{\alpha_{2}}(t)$ to see that $U_{\alpha_{2}}(t)=U_{\beta_{2}}\left(t^{q}\right)$ for all $t \in k$. Thus, $X$ contains a short root subgroup of $G$, as claimed.

Now let $\theta: G \rightarrow B_{n}$ be the morphism given in the proof of Lemma 2.1. Then $\theta(X)$ contains long root subgroups of $B_{n}$, and hence is determined by [25, Theorem 2.1]. We deduce that $\theta(X)=Y$ or $Y_{\tau}$ for some long subsystem subgroup $Y$ of $B_{n}$. Now we argue as in the proof of Lemma 2.1 that Theorem 1 holds.

\section{Lemma 2.7. Theorem 1 holds in case (v) of Table 9.}

Proof. Here $\left(X, L^{\prime}, G, p\right)=\left(B_{4}\right.$ or $\left.C_{4}, E_{6}, E_{7}, 2\right)$. We first argue that up to $G$-conjugacy, there are at most two closed complements to $Q$ in $Q X$. Recall that $\tilde{X} \leqslant L^{\prime}$ and $Q X=Q \tilde{X}$. By induction, $\tilde{X}=C_{4}$ and $\tilde{X}$ lies in a subgroup $F_{4}$ of $L^{\prime}$. We know that $\left.Q\right|_{F_{4}}=Q_{1} \oplus Q_{2}$ where $Q_{1}=V_{F_{4}}\left(\lambda_{4}\right)$ has dimension 26 and $\operatorname{dim} Q_{2}=1$. Then $\left.Q_{1}\right|_{\tilde{X}}=V_{C_{4}}\left(\lambda_{2}\right)$. Let $R$ be a $\tilde{X}$-module containing $Q$ with codimension 1 , such that $R / Q_{2}$ is indecomposable of dimension 27 (take $\left.R=Q_{2} \oplus W_{\tilde{X}}\left(\lambda_{2}\right)^{*}\right)$.

For $q$ a power of 2 , let $\tilde{X}(q) \simeq C_{4}(q)$ be the subgroup of $\mathbb{F}_{q}$-rational points in $\tilde{X}$. By $[\mathbf{1 8}], H^{1}\left(\tilde{X}(q), Q_{1}\right)$ has dimension 1 , and hence by Lemma 1.2 , $H^{1}(\tilde{X}(q), R)=0$. Then by Lemma $1.4, H^{1}\left(\tilde{X}, R^{(e)}\right)=0$ for all $e \geqslant 0$, and so by Lemma 1.3, all closed complements to $R$ in $R \tilde{X}$ are conjugate.

Fix $r \in R \backslash Q$. Then the classes of closed complements to $Q$ in $Q \tilde{X}$ are 
represented by $\tilde{X}$ and $\tilde{X}^{\lambda r}=\{\tilde{x}(\lambda r-\lambda(r \tilde{x})) \mid \tilde{x} \in \tilde{X}\}$ for $\lambda \in k^{*}$. If $T_{1}=Z(L)$, a torus of dimension 1, then $T_{1}$ induces the full group of scalars on $Q$, and therefore fuses all of the complements $\tilde{X}^{\lambda r}$ (for $\lambda \in k^{*}$ ). Hence there are at most two closed complements to $Q$ in $Q \tilde{X}$ which are not $G$-conjugate.

On the other hand, it is shown in $[25, \S 4]$, that $G$ has two conjugacy classes of subgroups $C_{4}$ containing long root subgroups. Take $X_{1}, X_{2}$ to be representatives of these classes, such that $X_{1}$ lies in an $E_{6}$ subgroup of $G$, while $X_{2}=Y_{\tau}$, where $Y$ is a subsystem subgroup $A_{7}$ and $\tau \in G$ is an involution inducing a graph automorphism on $Y$. Then $X_{2}$ centralizes the involution $\tau$, and hence lies in a parabolic subgroup $P_{0}$ of $G$. Now $P_{0}$ is not a $D_{n}$-type parabolic, since otherwise the proof of Lemma 2.5 would force $X_{2}=C_{4}$ to lie in a conjugate of a Levi subgroup $D_{n}$, which is impossible. Hence $P_{0}$ is an $E_{6}$ parabolic.

Thus both representatives $X_{1}$ and $X_{2}$ lie in an $E_{6}$ parabolic; it follows that there are precisely two closed complements to $Q$ in $Q \tilde{X}$ which are not $G$-conjugate, and hence are conjugate to $X_{1}$ and $X_{2}$. Hence $X$ is conjugate to $X_{1}$ or $X_{2}$, and is in conclusion (II) or (III) of Theorem 1.

\section{Lemma 2.8. Theorem 1 holds in case (vi) of Table 9.}

Proof. Here $\left(X, L^{\prime}, G, p\right)=\left(D_{4}, E_{6}, E_{7}, 2\right)$; also $\tilde{X}<F_{4}<L^{\prime}$ and is generated by short root subgroups of the $F_{4}$. Moreover $\left.Q\right|_{F_{4}}=Q_{1} \oplus Q_{2}$, where $\left.Q_{1}\right|_{F_{4}}=$ $V_{F_{4}}\left(\lambda_{4}\right)$ of dimension 26, and $\operatorname{dim} Q_{2}=1$. Then $\left.Q_{1}\right|_{\bar{X}}=V_{\bar{X}}\left(\lambda_{2}\right)$.

We show that conclusion (IV) of Theorem 1 holds under these circumstancesthere are infinitely many $P$-classes of complements to $Q$ in $Q \tilde{X} T_{1}$ (where $T_{1}=Z(L)$ ), and none of these classes are fused in $G$.

Suppose that $Y$ and $Z$ are complements to $Q$ in $Q \tilde{X}$ which are not $P$-conjugate to each other nor to $\tilde{X}$; we argue that $Y$ and $Z$ are not $G$-conjugate. First we claim that $C_{G}(Y)^{\circ}=C_{G}(Z)^{\circ}=Q_{2}$. For certainly $Q_{2} \leqslant C_{G}(Y)^{\circ}$, and if $C_{G}(Y)^{\circ}$ is not unipotent, then there is a 1-dimensional torus $T_{0}$ in $C_{G}(Y)^{\circ}$, normalizing $Q_{2}$. The group $Y Q_{2} T_{0}$ lies in a parabolic, say $P_{1}$. Then $Y Q_{2} \leqslant P_{1} \cap P$, and from [11, $\S 2.8$, we see that $P_{1}$ must be equal to $P$. Hence $Y \leqslant C_{P}\left(T_{0}\right)$, and so $Y$ lies in a $P$-conjugate of $L$, forcing $Y$ to be $P$-conjugate to $\tilde{X}$, a contradiction. Hence $C_{G}(Y)^{\circ}$ is unipotent. If $C_{G}(Y)^{\circ}>Q_{2}$, then there exists $u \in C_{G}(Y)^{\circ} \backslash Q_{2}$ normalizing $Q_{2}$. But $N_{G}\left(Q_{2}\right)=Q F_{4} T_{1}$ (as $N_{G}\left(Q_{2}\right)$ lies in a parabolic $P_{2}$ and contains $Q F_{4}$, so $P_{2}=P$ and $\left.N_{P}\left(Q_{2}\right)=Q F_{4} T_{1}\right)$. So $u \in C_{Q F_{4}}(Y)=Q_{2}$, a contradiction.

Thus we have established that $C_{G}(Y)^{\circ}=C_{G}(Z)^{\circ}=Q_{2}$. If $Y$ and $Z$ are $G$-conjugate, say $Z=Y^{g}$, then $C_{G}(Z)=C_{G}(Y)^{g}$, so $g \in N_{G}\left(Q_{2}\right)=Q F_{4} T_{1}<P$. Therefore $Z$ is $P$-conjugate to $Y$, contrary to assumption.

It remains to show that $P$ does indeed contain infinitely many classes of complements to $Q$ in $Q \tilde{X}$. For $q=2^{a}$, let $\tilde{X}(q)$ be the group of $\mathbb{F}_{q}$-rational points in $\widetilde{X}$. Observe that by [18], $H^{1}\left(\widetilde{X}(q), Q_{1}\right)$ has dimension 2 for all sufficiently large $q$. Hence by Lemma $1.4, H^{1}\left(\tilde{X}, Q_{1}\right)$ has dimension 2 also. If $\gamma: \widetilde{X} \rightarrow Q_{1}$ is a rational 1-cocycle, define

$$
\widetilde{X}_{\gamma}=\{\tilde{x} \gamma(\tilde{x}) \mid \tilde{x} \in \tilde{X}\},
$$

so that $\widetilde{X}_{\gamma}$ is a complement to $Q_{1}$ in $Q_{1} \tilde{X}$. Moreover, $\widetilde{X}_{\gamma}$ is closed (it is the image of the morphism $\tilde{x} \rightarrow \tilde{x} \gamma(\tilde{x}))$. Finally, since $H^{1}\left(\tilde{X}, Q_{1}\right)$ is 2-dimensional, not all the complements $\tilde{X}_{\gamma}$ are fused by $T_{1}=Z(L)$. The result follows. 
REMARK 2.9. Take $\left\{\gamma_{i} \mid 1 \leqslant i \leqslant 4\right\}$ and $\left\{\alpha_{j} \mid 1 \leqslant j \leqslant 7\right\}$ as bases of the root systems of $D_{4}$ and $E_{7}$ respectively. It is not hard to show that any complement to $Q$ in $Q \bar{X}$ is $G$-conjugate to $\tilde{X}_{(a, b)}=\left\langle x_{ \pm \gamma_{i}}(t) \mid t \in k, 1 \leqslant i \leqslant 4\right\rangle$, for $x_{ \pm \gamma_{i}}(t)$ as below and for some choice of $a, b \in k$ :

$$
\begin{aligned}
x_{\gamma_{1}}(t) & =x_{\alpha_{3}}(t) x_{\alpha_{5}}(t), \\
x_{-\gamma_{1}}(t) & =x_{-\alpha_{3}}(t) x_{-\alpha_{5}}(t), \\
x_{\gamma_{2}}(t) & =x_{\alpha_{1}}(t) x_{\alpha_{6}}(t), \\
x_{-\gamma_{2}}(t) & =x_{-\alpha_{1}}(t) x_{-\alpha_{6}}(t), \\
x_{\gamma_{3}}(t) & =x_{\alpha_{3}+\alpha_{4}}(t) x_{\alpha_{4}+\alpha_{5}}(t) x_{(1123211)}(a t), \\
x_{-\gamma_{3}}(t) & =x_{-\alpha_{3}-\alpha_{4}}(t) x_{-\alpha_{4}-\alpha_{5}}(t) x_{(1111111)}(a t), \\
x_{\gamma_{4}}(t) & =x_{\alpha_{2}+\alpha_{3}+\alpha_{4}}(t) x_{\alpha_{2}+\alpha_{4}+\alpha_{5}}(t) x_{(1223211)}(b t), \\
x_{-\gamma_{4}}(t) & =x_{-\alpha_{2}-\alpha_{3}-\alpha_{4}}(t) x_{-\alpha_{2}-\alpha_{4}-\alpha_{5}}(t) x_{(1011111)}(b t) .
\end{aligned}
$$

The proof of Theorem 1 is now complete.

\section{Proof of Theorem 2}

Let $X$ and $G$ be as in Theorem 2. Let $l$ be the untwisted rank of $X$ and $n=\operatorname{rank}(G)$, so that $l>\frac{1}{2} n$. We will assume throughout that $p \neq 2$ when $X$ has type $B_{n}$. Let $\sigma$ be a Frobenius morphism of $G$. We assume that $\sigma$ fixes the conjugacy class of long root subgroups of $G$. (Observe that this is the case if $X<G_{\sigma}$ : for, suppose this is not true, so that $G_{\sigma}$ is of type ${ }^{2} F_{4},{ }^{2} G_{2}$ or ${ }^{2} B_{2}$. We may assume that $X$ is not of the same type as $G_{\sigma}$ (otherwise take $\bar{X}=G$ to obtain (I) of Theorem 2). For types ${ }^{2} F_{4},{ }^{2} B_{2}$, the $r$-rank of $G_{\sigma}$ is at most 2 or 1 respectively, for odd primes $r$ (see $[\mathbf{1 5}, 10.2]$ ); whereas for suitable $r$, the $r$-rank of $X$ is greater than this; and for type ${ }^{2} G_{2}$, the centralizer of an involution $t$ in $G_{\sigma}$ is $\langle t\rangle \times L_{2}(q)$, which cannot contain $C_{X}(t)$ when $t \in X$.)

It is convenient to begin with a lemma which takes care of the $\sigma$-stability of the subgroups $\bar{X}$ to be produced in conclusion (I) of Theorem 2 .

LEMMA 3.1. Let $\bar{X}$ be a simple closed connected subgroup of $G$ with $\operatorname{rank}(\bar{X})>$ $\frac{1}{2} \operatorname{rank}(G)$, and let $\delta$ be a Frobenius morphism of $\bar{X}$ such that $X=O^{p}\left(\bar{X}_{\delta}\right)$ is a finite quasisimple group of Lie type over $\mathbb{F}_{q}$ with $q>2$. Suppose that $X<G_{\sigma}$. Then $\bar{X}$ is $\sigma$-stable.

Proof. The possibilities for $\bar{X}$ are given by Theorem 1 . The result is trivial if $\bar{X}=G$, so take $\bar{X}<G$.

Suppose first that $\bar{X}$ contains a long root subgroup $U$ of $G$ such that $U \cap X \neq 1$. Let $1 \neq u \in U \cap X$. Then $u=u^{\sigma}$, so $U \cap U^{\sigma} \neq 1$. By assumption, $U^{\sigma}$ is a long root group, so by $[25,1.1(i i)], U=U^{\sigma}$. Since $\bar{X}=\langle X, U\rangle, \bar{X}$ is $\sigma$-stable.

Thus we now assume that $\bar{X}$ contains no long root subgroup $U$ with $U \cap X \neq 1$. 
By inspection using Theorem 1, the possibilities for $(X, G)$ are as shown in Table 10.

TABLE 10

\begin{tabular}{lllll}
\hline & $X=O^{p^{\prime}}\left(\bar{X}_{\delta}\right)$ & \multicolumn{1}{c}{$G$} & $p$ & \multicolumn{1}{c}{ Comment } \\
\hline$(1)$ & $D_{l}^{\varepsilon}(q)$ & $A_{2 l-1}$ & & $\bar{X}$ a Levi complement \\
$(2)$ & $A_{l}^{\varepsilon}(q)$ & $C_{n}$ & & \\
$(3)$ & $A_{2}^{\varepsilon}(q)$ & $B_{3}$ & 3 & \\
$(4)$ & $G_{2}(q)$ & $C_{3}$ & 2 & \\
$(5)$ & ${ }^{2} G_{2}(q)$ & $B_{3}$ & 3 & \\
$(6)$ & ${ }^{2} B_{2}(q)$ & $B_{3}$ or $C_{3}$ & 2 & \\
$(7)$ & $D_{l}^{\varepsilon}(q)$ & $C_{n}$ & 2 & $\bar{X}$ generated by short root subgroups of $G$ \\
$(8)$ & $B_{1-1}(q)$ & $C_{n}$ & 2 & $\bar{X}<D_{1}$ of $(7)$ \\
$(9)$ & $D_{4}^{\varepsilon}(q)$ & $F_{4}, E_{6}, E_{7}$ & 2 & $\bar{X}<F_{4} \leqslant G$, \\
& & & & $\bar{X}$ generated by short root subgroups of $F_{4}$ \\
$(10)$ & $D_{4}^{\varepsilon}(q)$ & $E_{7}$ & 2 & $\bar{X}$ as in $($ IV) of Theorem 1 \\
$(11)$ & $B_{3}(q), A_{3}(q)$ & $F_{4}$ & 2 & $\bar{X}<D_{4}$ of $(9)$ \\
$(12)$ & $D_{4}^{\varepsilon}(q)$ & $E_{7}$ & & $\bar{X}<A_{7}<G$ \\
$(13)$ & ${ }^{2} F_{4}(q)$ & $F_{4}, E_{6}, E_{7}$ & 2 & \\
\hline
\end{tabular}

For cases (7), (8) and (11), $\bar{X}$ contains short root subgroups of $G$. If $G^{*}$ is the dual group of $G$, there is a morphism $\phi: G \rightarrow G^{*}$ as given in the proof of Lemma 2.1. Then $\phi(\bar{X})$ contains long root subgroups of $G^{*}$ intersecting $\phi(X)$ nontrivially. Moreover, there is a Frobenius morphism $\sigma^{\prime}$ of $G^{*}$ such that $\phi \sigma=\sigma^{\prime} \phi$. By the second paragraph of this proof, $\phi(\bar{X})$ is $\sigma^{\prime}$-stable, and hence $\bar{X}$ is $\sigma$-stable.

Next, observe that in case (1), $X$ fixes a unique quadratic form on the natural module for $G=\mathrm{SL}_{2 l}$, so $X$ lies in a unique subgroup $D_{l}$ of $G$. This subgroup must therefore be $\sigma$-stable.

We are left with cases (2)-(6), (9), (10), (12) and (13) of the table. Notice that in all these cases except $(10), N_{G}(\bar{X})$ induces the full (algebraic) automorphism group $\operatorname{Aut}(\bar{X})$ on $\bar{X}$. (See $[\mathbf{1 3}, 2.15]$ for this fact for (12); the remaining cases are clear.)

We will argue that in cases (2)-(6), (9), (12) and (13),

$$
N_{G}(X) \leqslant N_{G}(\bar{X}) \text {. }
$$

Suppose for the moment that $(\ddagger)$ holds. Consider the subgroup $\bar{X}^{\sigma}$. By Theorem 1 , this is $G$-conjugate to $\bar{X}$. (Note that when $G=E_{7}$ and $\bar{X}=D_{4}<F_{4}<G$, we have $\bar{X}^{\sigma}<F_{4}^{\sigma} \cong F_{4}$, so $\bar{X}^{\sigma}$ is conjugate to $\bar{X}$; a similar result holds when $\bar{X}=D_{4}<A_{7}<G=E_{7}$.) Therefore $\bar{X}^{\sigma}=\bar{X}^{g}$ for some $g \in G$. Then $X$ and $X^{g^{-1}}$ both lie in $\bar{X}$. By $[25,5.1]$, it follows that $X^{g^{-1}}=X^{n}$ for some $n \in N_{G}(\bar{X})$. Then $n g \in N_{G}(X)$. By $(\ddagger), n g \in N_{G}(\bar{X})$, so $g \in N_{G}(\bar{X})$, proving that $\bar{X}^{\sigma}=\bar{X}^{g}=\bar{X}$, as required.

So it remains to prove ( $\ddagger)$ for cases (2)-(6), (9), (12) and (13) (and to deal with case (10)). This is clear for the irreducible embeddings (3)-(5): $C_{G}(X)=1$, and any automorphism induced by $N_{G}(X)$ must fix the high weight of $\left.V\right|_{X}$, and hence lie in $N_{G}(\bar{X})$. 
Now consider (2). Let $V$ be the natural $2 n$-dimensional module for $C_{n}$. Then $\left.V\right|_{\bar{X}}=V\left(\lambda_{1}\right) \oplus V\left(\lambda_{l}\right) \oplus V(0)^{2 n-2 l-2}$. Then $N_{G}(X)$ stabilizes $V\left(\lambda_{1}\right) \oplus V\left(\lambda_{l}\right)$. But the full stabilizer of $V\left(\lambda_{1}\right) \oplus V\left(\lambda_{l}\right)$ in $G$ is $N_{G}(\bar{X})$.

Now consider (6). Applying the morphism $B_{3} \rightarrow C_{3}$ of Lemma 2.1 if necessary, we assume $G=C_{3}$. If $\bar{X}=C_{2}$, a Levi subgroup of $C_{3}$, then $C_{G}(X)=C_{G}(\bar{X})=$ $A_{1}$, and $N_{G}(X) \leqslant N_{G}\left(A_{1}\right)=N_{G}(\bar{X})$, giving $(\dagger)$. And if $\bar{X}=B_{2}$ generated by short root groups in $C_{3}$, then $X$ and $\bar{X}$ act indecomposably on the natural 6-dimensional module for $C_{3}$ (note that $H^{1}\left(X, V_{X}\left(\lambda_{1}\right)\right)$ has dimension 1 by [18]), so $C_{G}(X)=$ $C_{G}(\bar{X})$ and $(\ddagger)$ holds again.

Consider (12). Pick a fundamental subgroup $A<X$ with $A \simeq \mathrm{SL}_{2}(q)$. Then there is a subgroup $\mathrm{SO}_{4}$ of $A_{7}$ such that $A$ lies in one of the factors $\mathrm{SL}_{2}$ of $\mathrm{SO}_{4}$. So there are commuting subgroups $J_{1}, J_{2}$, each $J_{i}$ a fundamental $A_{1}$, such that $A_{1} \times A_{1} \simeq J_{1} \times J_{2}<A_{7}$ and $A$ lies in a diagonal subgroup $\bar{A}=\mathrm{SL}_{2}(k)$ of $J_{1} \times J_{2}$. Now $C_{G}\left(J_{1} J_{2}\right)=J_{3} D_{4}$ where $J_{3} \simeq A_{1}$. Further, $N_{G}\left(J_{3}\right)=J_{3} D_{6}$. As a subgroup of $D_{6}$, $J_{1} J_{2}=\mathrm{SO}_{4}$. On the 4-space $A$ and $\bar{A}$ act as $\mathrm{SL}_{2}$ on $V_{2} \otimes V_{2}$. For $p \neq 2$ this breaks up as $V_{3} \oplus V_{1}$ (where $V_{i}$ is an irreducible $i$-dimensional $\mathrm{SL}_{2}$-module); and for $p=2$ $(q>2)$, it is indecomposable $V_{1} / V_{2} / V_{1}$. Hence $C_{D_{6}}(A)=C_{D_{0}}(\bar{A})=B_{4}$, and so $C_{G}(\vec{A}) \geqslant J_{3} B_{4}$. Pick $t \in A$ of order 2 if $p \neq 2$, and of order $q+1$ if $p=2$. For $p \neq 2, C_{G}(t)=J_{3} D_{6}$ (note that if $\left\langle e_{i}\right\rangle=Z\left(J_{i}\right)$, then $t=e_{1} e_{2}=e_{3}$ ), so as $C_{G}(A) \leqslant$ $C_{G}(t)$, we have $C_{G}(A)=C_{G}(\bar{A})=J_{3} B_{4}$. For $p=2, C_{G}(t)$ contains $J_{3} B_{4}$, so must be $J_{3} D_{5} T_{1} \leqslant J_{3} D_{6}$. So in any case,

$$
C_{G}(A)=C_{G}(\bar{A})=J_{3} B_{4} .
$$

Notice also that $N_{G}(A) \leqslant J_{3} D_{6}$ (anything in $N_{G}(A)$ normalizes $\langle t\rangle$, if $p \neq 2$, or some Sylow $r$-subgroup where $r \mid q+1$, if $p=2)$, so $N_{G}(A)=A C_{G}(A)$ and $N_{G}(\bar{A})=\bar{A} C_{G}(A)$. Since $X, \bar{X}$ are generated by $X$-conjugates of $A, \bar{A}$ respectively, we deduce that $C_{G}(X)=C_{G}(\bar{X})$. Moreover $N_{G}(\bar{X})$ induces $\bar{X} S_{3}$ on $\bar{X}$, and hence induces $X N_{S_{3}}(X)$ on $X$. If $n \in N_{G}(X)$ induces a further outer automorphism on $X$, this may be taken to be a field automorphism $\phi$; but then some $n$ will induce $\phi$ on $A$, whereas $N_{G}(A)=A J_{3} B_{4}$ induces no field automorphism on $A$. Thus $N_{G}(X) \leqslant N_{G}(\bar{X})$, which proves ( $\left.\ddagger\right)$ for the embedding (12).

Next consider (9). Choose a subgroup $A=\operatorname{SL}_{3}^{\delta}(q)$ of $X$ with $Z(A)=\langle t\rangle$ where $o(t)=3$. Then $A$ is generated by short root elements of the $F_{4}$ containing $X$, and there are commuting long and short root subsystem subgroups $A_{2}$ and $\bar{A}_{2}$ (respectively) such that $A<\tilde{A}_{2}<\bar{X}=D_{4}$ and $\langle t\rangle=Z\left(A_{2} \tilde{A}_{2}\right)$. Further, $C_{G}(t)$ is $A_{2} \widetilde{A}_{2}\left(G=F_{4}\right), \quad\left(A_{2} A_{2} A_{2}\right)\left\langle w_{3}\right\rangle\left(G=E_{6}\right)$ or $A_{2} A_{5} \quad\left(G=E_{7}\right)$, where $w_{3}$ is an element of order 3 in $W(G)$. The subgroup $\tilde{A}_{2}$ is embedded in $A_{2} A_{2}$ (in $E_{6}$ ) as a diagonal subgroup $\left\{(g, g) \mid g \in A_{2}\right\}$. Since $C_{G}\left(\tilde{A}_{2}\right) \leqslant C_{G}(A) \leqslant C_{G}(t)$, an easy calculation in $C_{G}(t)$ shows that $C_{G}(A)=C_{G}\left(\tilde{A}_{2}\right)$. Hence $C_{G}(X)=C_{G}(\bar{X})$. As in the previous paragraph, we deduce that $N_{G}(X) \leqslant N_{G}(\bar{X})$.

We now consider (13). There is an element $t$ of order 3 such that $C_{X}(t)=A=$ $\mathrm{SU}_{3}(q)$ (see [14]). Then $C_{F_{4}}(t)=A_{2} \tilde{A}_{2}$, and there is a diagonal subgroup $\bar{A} \simeq A_{2}$ with $A<\bar{A}<A_{2} \bar{A}_{2}$. As above, $C_{G}(\bar{A}) \leqslant C_{G}(A) \leqslant C_{G}(t)$, which is $A_{2} \bar{A}_{2}$, $A_{2} A_{2} A_{2}\left\langle w_{3}\right\rangle$ or $A_{2} A_{5}$. Calculating in $C_{G}(t)$, we see that $C_{G}(X)=C_{G}(\bar{X})$, and hence as usual $N_{G}(X) \leqslant N_{G}(\bar{X})$.

It remains to consider case (10). Here $X<Q D<Q L$, where $D<L^{\prime}=E_{6}$, $D \simeq D_{4}$. We saw in Lemma 2.8 that $H^{1}(D, Q)$ has dimension 2 . By $[12,7.1]$, the restriction map $H^{\prime}(D, Q) \rightarrow H^{1}\left(D\left(q^{\prime}\right), Q\right)$ is an isomorphism for large $q^{\prime}$ (where $\left.D\left(q^{\prime}\right) \simeq D_{4}\left(q^{\prime}\right)\right)$. Now choose a (possibly twisted) subgroup $D^{\varepsilon}(q) \simeq X$ of $D$ with $Q X=Q D^{\varepsilon}(q)$, and choose a suitably large $q^{\prime}$ and $D\left(q^{\prime}\right)$ with $D^{\varepsilon}(q)<D\left(q^{\prime}\right)$. 
By [18], $H^{1}\left(D^{\varepsilon}(q), Q\right)$ and $H^{1}\left(D\left(q^{\prime}\right), Q\right)$ both have dimension 2, so we conclude that the restriction $H^{1}(D, Q) \rightarrow H^{1}\left(D^{\varepsilon}(q), Q\right)$ is an isomorphism.

Now we have $X=\left\{d \gamma(d) \mid d \in D^{\varepsilon}(q)\right\}$ for some 1-cocycle $\gamma: D^{\varepsilon}(q) \rightarrow Q$. From the above observation, $\gamma$ is the restriction of a rational 1 -cocycle $\bar{\gamma}: D \rightarrow Q$, and $\bar{\gamma}$ is determined up to 1 -coboundaries. Therefore $X$ lies in the closed complement $\bar{X}=\{d \bar{\gamma}(d) \mid d \in D\}$ to $Q$ in $Q D$, and the closed complements containing $X$ are $C_{Q}\left(D^{\varepsilon}(q)\right)$-conjugate. Since $C_{Q}\left(D^{\varepsilon}(q)\right)=C_{Q}(D)=C_{Q}(\bar{X})$, we deduce that $\bar{X}$ is the unique complement containing $X$, and hence is $\sigma$-stable.

This completes the proof.

We now proceed with the proof of Theorem 2 by induction on $\operatorname{dim} G$.

LEMMA 3.2. Theorem 2 holds if $X$ lies in a proper connected reductive subgroup of $G$.

Proof. Suppose $X \leqslant H<G$ with $H$ connected and reductive. As in the proof of Lemma 2.2, $X$ lies in one of the simple factors of $H^{\prime}$, so we may assume that $H$ is simple.

By induction, $X$ lies in a suitable connected simple subgroup $\bar{X}$ of $H$; moreover, if $X<G_{\sigma}$, then $\bar{X}$ is $\sigma$-stable, by Lemma 3.1. Then $X=O^{p^{\prime}}\left(\bar{X}_{\delta}\right)$, for some $\delta$, by [25, 5.1]. Hence (I) of Theorem 2 holds.

In view of Lemma 3.2, we assume that

$X$ lies in no proper connected reductive subgroup of $G$.

In particular, we may take it that $Z(X)=1$, so that $X$ is simple.

\section{Lemma 3.3. Suppose $G$ is a classical group. Then Theorem 2 holds.}

Proof. Let $V$ be the natural module for $G$ (if $p=2$ and $G=B_{n}$, take $V$ to be the natural $2 n$-dimensional module). If $X$ is irreducible on $V$, then by [32], $X$ lies in a simple connected subgroup $\bar{X}$ of $G$ of the same type as $X$, contrary to ( $\dagger$ ).

Thus $X$ is reducible on $V$, and so by $(\dagger), X$ lies in a proper parabolic subgroup $P=Q L$ of $G$. Choose $P$ minimal. Arguing by induction (as in the derivation of Table 9 in the proof of Theorem 1), we see that either $X$ and $L^{\prime}$ have the same type (that is, $X \simeq O^{p^{\prime}}\left(L_{\delta}\right)$ for some $\delta$ ) or $\left(X, L^{\prime}\right)=\left(B_{l}(q), D_{l+1}\right),\left(D_{l}^{\varepsilon}(q), B_{l}\right)$ or $p=2$ and $\left(X, L^{\prime}\right)=\left(D_{l}^{\varepsilon}(q), C_{l}\right)$. As $G$ is classical, the high weights for $L^{\prime}$ which occur in $Q$ are

$$
\begin{aligned}
2 \lambda_{1}, \lambda_{1}, \lambda_{2} & \text { if } L^{\prime}=A_{l} \text { (and } 2 \lambda_{1} \text { occurs only if } G=C_{n} \text { ), } \\
\lambda_{1} & \text { if } L^{\prime} \neq A_{l} .
\end{aligned}
$$

By $(\dagger), X$ lies in no conjugate of $L^{\prime}$, and hence $H^{1}(X, W) \neq 0$ for some irreducible $L^{\prime}$-module $W=V_{L^{\prime}}(\lambda)$ occurring in $Q$. Therefore we deduce from Lemma 1.8 that one of the possibilities listed in Table 11 holds.

TABLE 11

\begin{tabular}{rlllll}
\hline & $X$ & $\lambda$ & $L^{\prime}$ & $G$ & $p$ \\
\hline (i) & $C_{l}(q)$ & $\lambda_{1}$ & $C_{l}$ & $C_{n}$ & 2 \\
(ii) & $C_{l}(q)$ & $\lambda_{1}$ & $D_{l+1}$ & $D_{n}$ & 2 \\
(iii) & $B_{2}(3)$ & $\lambda_{1}$ & $B_{2}$ & $B_{3}$ & 3 \\
(iv) & ${ }^{2} A_{2}(3)$ & $2 \lambda_{1}$ & $A_{2}$ & $C_{3}$ & 3 \\
\hline
\end{tabular}


In case (i) with $l>2$, we argue as in the proof of Lemma 2.6 that some root subgroup of $X$ lies in a short root subgroup of $G$. If $\theta: G \rightarrow B_{n}$ is the morphism given in the proof of Lemma 2.1, then some root subgroup of $\theta(X)$ lies in a long root subgroup of $B_{n}$, and so Theorem 2 holds by $[25,6.1]$.

Now consider case (i) with $l=2$. Here $\left(X, L^{\prime}, G, p\right)=\left(C_{2}(q), C_{2}, C_{3}, 2\right)$ and $Q$ is the usual 5-dimensional indecomposable orthogonal module for $X$. There is a 6-dimensional indecomposable $k X$-module $R$ containing $Q$ (take $R$ to be the usual 6-dimensional module for a 6-dimensional orthogonal group containing $X$ ). Fix $v \in R \backslash Q$. Since $H^{1}\left(X, V_{X}\left(\lambda_{2}\right)\right)$ is 1-dimensional, the complements to $Q$ in $Q X$ which are not conjugate to $X$ are conjugates of $X^{\lambda v}=\{x(\lambda v-\lambda x(v)) \mid x \in X\}$ for $\lambda \in k^{*}$. If $T_{1}=Z(L)$, a torus of dimension 1 , then $T_{1}$ induces the full group of scalars on $Q$, and therefore fuses all the complements $X^{\lambda v}\left(\lambda \in k^{*}\right)$. Hence $Q X$ contains at most two complements to $Q$ which are not $G$-conjugate. Now observe that there are indeed two non-conjugate complements: one lies in $L^{\prime}$, and the other in $Y_{\tau}$, where $Y$ is a short root subsystem subgroup of type $D_{3}$ in $G$. They are non-conjugate as $L^{\prime}$ fixes a 2-space on the natural 6-dimensional module for $G$, whereas $Y_{\tau}$ does not. Hence Theorem 2 holds in this case.

Next suppose case (ii) holds, so that $\left(X, L^{\prime}, G, p\right)=\left(B_{l}(q), D_{l+1}, D_{n}, 2\right)$. Here the $L^{\prime}$-composition factor $W$ in $Q$ is $W=V_{L^{\prime}}\left(\lambda_{1}\right)$, the natural $(2 l+2)$-dimensional module for $L^{\prime}=D_{l+1}$. Then $\left.W\right|_{X}$ is indecomposable, with factors $V_{X}(0) / V_{X}\left(\lambda_{1}\right) / V_{X}(0)$. By Lemma $1.8, H^{1}\left(X, V_{X}\left(\lambda_{1}\right)\right)$ has dimension 1 . Hence Lemma 1.2 gives $H^{1}(X, W)=0$ in fact, which is a contradiction.

Now consider case (iii), in which $\left(X, L^{\prime}, G, p\right)=\left(B_{2}(3), B_{2}, B_{3}, 3\right)$. We argue that (II) of Theorem 2 holds; that is, $G$ contains a subgroup $X=B_{2}(3)$ acting indecomposably on $V=V_{G}\left(\lambda_{1}\right)$ and $X$ is unique up to $G$-conjugacy. Let $Y$ be a subgroup $B_{2}(3)$ of $L^{\prime}$. Since $H^{1}(Y, Q)$ has dimension 1 by Lemma 1.8 , we can choose $X$ to be a complement to $Q$ in $Q Y$ which is not $Q$-conjugate to $Y$. Let $v \in V$ be a non-zero singular vector fixed by $P=Q L$. Observe that $Q$ acts transitively on the set of non-degenerate 2-spaces containing $v$. If $X$ fixes one of these 2-spaces, it follows that $X$ is $Q$-conjugate to $Y$, contrary to assumption. Therefore $X$ acts indecomposably on $V$. As in the argument of case (i) with $l=2$, the torus $T_{1}=Z(L)$ fuses all the complements in $Q Y$ not conjugate to $Y$, and so we deduce that $X$ is unique up to $G$-conjugacy.

It remains to deal with case (iv). Let $Y$ be a subgroup ${ }^{2} A_{2}(3)$ of $L^{\prime}$ such that $Q Y=Q X$. By Lemma 1.7, $Q Y$ contains a complement to $Q$ which is not $Q$-conjugate to $Y$; take $X$ to be this complement. Then $X$ acts indecomposably on the usual 6-dimensional module for $G$, so $X$ is not $G$-conjugate to $Y$. Now argue as in the previous paragraph that $X$ is unique up to $G$-conjugacy.

\section{Lemma 3.4. Theorem 2 holds if $G=F_{4}$.}

Proof. Here $X=X(q)$, for $q>2$, a group of untwisted rank $l \geqslant 3$. First consider the case where $q$ is odd and $l=3$. Let $V=V_{F_{4}}\left(\lambda_{4}\right)$; so $\operatorname{dim} V=26$ if $p \neq 3$ and $\operatorname{dim} V=25$ if $p=3$. We consider the action of $X$ on $V$. Lemma 1.11 gives the possible high weights $\lambda$ (up to group automorphism) for which $V_{X}(\lambda)$ can occur as a composition factor of $\left.V\right|_{X}$. (Note that for $X=A_{3}^{\varepsilon}(q)$ or $C_{3}(q), V_{X}\left(\lambda_{1}\right)$ is a module for the simple group only when $p=2$.) We apply Lemmas 1.2 and 1.8, 
noting that $\left.V\right|_{F_{4}}$ is self-dual, to conclude that either $X$ fixes a point on $V$ or $V^{*}$, or $X$ and the composition factors of $\left.V\right|_{X}$ are among the following (again, high weights are given up to automorphisms of $X$ ):

$$
\begin{gathered}
X=A_{3}^{\varepsilon}(q),\left.\quad V\right|_{X}=V\left(2 \lambda_{2}\right) / V\left(\lambda_{2}\right), \\
\quad \text { or } V\left(2 \lambda_{1}\right) / V\left(2 \lambda_{3}\right) / V\left(\lambda_{2}\right), p>3, \\
X=B_{3}(q),\left.\quad V\right|_{X}=V\left(2 \lambda_{1}\right), p=7 .
\end{gathered}
$$

Note that the stabilizers in $G$ of points in $V$ have positive dimension. Therefore, if $X$ fixes a point on $V, X$ lies in a proper closed connected subgroup $M$ of $G$. By $(\dagger), M$ lies in a parabolic subgroup $Q L$, with unipotent radical $Q$ and Levi factor $L$, where $L^{\prime}$ is of type $B_{3}$ or $C_{3}$.

By Lemma 3.3, we know the embedding of $X Q / Q$ in $L^{\prime} Q / Q$; in particular, the non-trivial $X$ composition factors in $Q$ have possible high weights $\lambda_{1}, \lambda_{2}$ or $\lambda_{3}$ if $X=A_{3}^{\varepsilon}(q), \lambda_{1}$ or $\lambda_{3}$ if $X=B_{3}(q)$ and $\lambda_{3}$ if $X=C_{3}(q)$. Since by Lemma 1.8, $H^{1}\left(X, V_{X}(\lambda)\right)=0$ for all these high weights $\lambda, X$ lies in a conjugate of $L^{\prime}$, contrary to (†). Thus, in fact, $X$ does not fix a point on $V$; so (i) or (ii) above holds.

In case (ii), where $X=B_{3}(q)$ and $\left.V\right|_{X}=V_{X}\left(2 \lambda_{1}\right)$, let $t$ be an involution in $X$ fixing a 5-dimensional subspace of the natural $k X$-module $W$. Note that $\left.S^{2} W\right|_{X}=V_{X}\left(2 \lambda_{1}\right) / V(0)^{2}$, and $\operatorname{dim} C_{S^{2} w}(t)=18$, so $\operatorname{dim} C_{V}(t)=16$, contradicting Lemma 1.12 .

In case (i), where $X=A_{3}^{\varepsilon}(q)$, let $W$ be the natural $k X$-module and let $t$ and $u$ be involutions in $X$ acting on $W$ as $\operatorname{diag}(i, i,-i,-i)$ and $\operatorname{diag}(1,1,-1,-1)$, respectively, relative to some basis of $W$ (where $i$ is a primitive fourth root of 1). Then $\operatorname{dim} C_{V\left(\lambda_{2}\right)}(t)=4, \quad \operatorname{dim} C_{V\left(2 \lambda_{1}\right)}(t)=4, \quad \operatorname{dim} C_{V\left(2 \lambda_{1}\right)}(u)=6$, and $\operatorname{dim} C_{V\left(2 \lambda_{2}\right)}(t)=12$ (11 for $p=3$ ). Again apply Lemma 1.12 to rule out both the configurations of (i). Thus the lemma holds when $X$ is of untwisted rank 3 with $q$ odd.

Now suppose $X=C_{3}(q)$ or $A_{3}(q)$ with $q$ even. Let $A=A_{2}(q)$ be a Levi factor of $X$. Then there exists a parabolic $P=L Q$ of $G$ with $A \leqslant P$. Taking $P$ minimal and applying Lemma 3.3, we have that $L^{\prime}$ is of type $A_{2}$. By Lemma $1.8, A$ is conjugate to a subgroup of $L^{\prime}$. By Lemma 3.3, $A=O^{p^{\prime}}\left(L_{\delta}^{\prime}\right)$, for some Frobenius morphism $\delta$ of $L^{\prime}$, and hence long root subgroups of $A$ lie in either long or short root subgroups of $G$. If they lie in long root subgroups, then $X$ is determined by $[25,6.1]$, so the conclusion of Theorem 2 holds. Otherwise, let $\theta: G \rightarrow G$ be the morphism of [36, Theorem 28] which interchanges long and short root groups of $G$. Then $\theta(X)$ is determined by $[\mathbf{2 5}, 6.1]$ and Theorem 2 again holds.

To finish the case where $l$ is 3 , suppose $X={ }^{2} A_{3}(q)$ with $q$ even. Let $x \in X$ be an element of order $q+1$ centralized by ${ }^{2} A_{2}(q)$. Since $q+1>3, C_{G}(x)^{\circ} \leqslant B_{3} T_{1}$ or $C_{3} T_{1}$ (see $[15,14.1]$ ). Then by Lemma 3.3, long root subgroups of $X$ lie in long or short root subgroups of $C_{G}(x)^{\circ}$ and the conclusion follows as above.

Now let $l \geqslant 4$. If $X=F_{4}^{\varepsilon}(q), 5.1$ of [25] gives the result. So assume $X \neq F_{4}^{\varepsilon}(q)$. 
Suppose $X \neq{ }^{3} D_{4}(q)$ and choose a subgroup $X_{3}$ in $X$ of untwisted Lie rank 3 over $\mathbb{F}_{q}$, such that root subgroups of $X_{3}$ are root subgroups of $X$. Then by the $l=3$ case, a root subgroup of $X_{3}$ lies in a root subgroup of $G$. So root subgroups of $X$ (or $\theta(X)$, with $\theta$ as above) are contained in long root subgroups of $G$ and the conclusion follows from $[25,6.1]$.

Finally, suppose $X={ }^{3} D_{4}(q)$. Let $V=V_{G}\left(\lambda_{4}\right)$. If $\left.V\right|_{X}$ is reducible, then by Lemma 1.11 all non-trivial composition factors of $\left.V\right|_{X}$ are 8-dimensional and so by Lemmas 1.2 and 1.8, $X$ fixes a point on $V$ and therefore lies in a proper connected reductive subgroup of $G$, contrary to $(\dagger)$. Therefore, $p=2$ and $\left.V\right|_{X}=V_{X}\left(\lambda_{2}\right)$ (up to an automorphism of $X$ ). By [14], there exists $t \in X$ of order $q^{2}+q+1$ such that $C_{X}(t) \geqslant A$, with $A \simeq A_{2}(q)$. But $C_{G}(t) \leqslant B_{3} T_{1}$ or $C_{3} T_{1}$ and the conclusion follows as before.

Lemma 3.5. Theorem 2 holds if $G=E_{6}$ and $X$ has untwisted rank 4. Moreover, if $X \neq D_{4}^{\varepsilon}(q)$, then long root subgroups of $X$ lie in long root subgroups of $G$.

Proof. Assume first that $p \neq 2$ and $X=C_{4}(q)$ or $B_{4}(q)$. Then there exists an involution $t \in X$ such that $C_{X}(t) \geqslant C_{3}(q)$ or $D_{4}^{\varepsilon}(q)$, respectively. Then $C_{X}(t)<$ $C_{G}(t)=A_{1} A_{5}$ or $T_{1} D_{5}$, respectively. So we have $D_{4}^{\varepsilon}(q)<T_{1} D_{5}$ or $C_{3}(q)<A_{1} A_{5}$. By Lemma 3.3, $X$ contains long root elements of $G$ and the result follows from $[25,6.1]$.

Using Lemmas 1.2, 1.8 and 1.11, we check that in the remaining cases $X$ fixes a point on $V_{G}\left(\lambda_{1}\right)$ or its dual. Thus $X$ lies in a point stabilizer, a proper subgroup of $G$ of positive dimension; hence by $(\dagger), X$ lies in a parabolic subgroup $P=Q L$. If $X=C_{4}(q)$ with $p=2$, then $L^{\prime}$ is of type $D_{5}$ and $Q$ is a spin module for $L^{\prime}$. This observation, together with Lemma 1.5, provides the following list of all possible high weights $\lambda$ of non-trivial $X$ composition factors in $Q$ :

\begin{tabular}{ll}
\hline \multicolumn{1}{c}{$X$} & \multicolumn{1}{c}{$\lambda$} \\
\hline$A_{4}^{\varepsilon}(q)$ & $\lambda_{1}, \lambda_{2}, \lambda_{3}, \lambda_{4}$ \\
$C_{4}(q), p=2$ & $\lambda_{4}$ \\
$D_{4}^{\varepsilon}(q)$ & $\lambda_{1}, \lambda_{3}, \lambda_{4}$ \\
\hline
\end{tabular}

Then by Lemma 1.8, $X$ lies in a conjugate of $L^{\prime}$, contrary to (†). The last statement of the lemma follows by inspection of the conclusion of Theorem 2 .

Lemma 3.6. Suppose $G=E_{7}$ and $X$ has untwisted rank 4. If $X$ does not fix a point on the 56-dimensional module $V_{G}\left(\lambda_{7}\right)$, then the conclusion of Theorem 2 holds. Moreover, if $X \neq D_{4}^{\varepsilon}(q)$, then long root subgroups of $X$ lie in long root subgroups of $G$.

Proof. Let $V=V_{E_{7}}\left(\lambda_{7}\right)$. The possible sets of composition factors of $\left.V\right|_{X}$ can be listed using Lemma 1.11. Since $X$ fixes no point on $V$, Lemma 1.8 together with Lemma 1.2 and the self-duality of $V$ forces $\left.V\right|_{X}$ to be one of the following (with 
the high weights given up to automorphisms of $X$ ):

(i) $\quad X=A_{4}^{\varepsilon}(q),\left.\quad V\right|_{X}=V_{X}\left(\lambda_{i}\right) \otimes V_{X}\left(p^{a} \lambda_{j}\right) / V_{X}\left(\lambda_{k}\right) \otimes V_{X}\left(p^{b} \lambda_{m}\right) / V_{X}(0)^{6}$,

$$
\begin{aligned}
& \quad i, j, k, m \in\{1,4\}, a, b \geqslant 1, \\
& V_{X}\left(\lambda_{1}+\lambda_{4}\right)^{2} / V_{X}\left(\lambda_{1}\right)^{2}, p=5, \\
& V_{X}\left(\lambda_{1}+\lambda_{4}\right)^{2} / V_{X}(0)^{8}, q=3,
\end{aligned}
$$

(ii) $\quad X=C_{4}(q),\left.\quad V\right|_{X}=V_{X}\left(\lambda_{3}\right) / V_{X}\left(\lambda_{1}\right), p \neq 3$,

$$
\begin{aligned}
& V_{X}\left(\lambda_{3}\right) / V_{X}\left(\lambda_{1}\right)^{2}, p=3 \\
& V_{X}\left(\lambda_{4}\right)^{i} / V_{X}\left(\lambda_{1}\right)^{j}, 2 i+j=7, p=2
\end{aligned}
$$

(iii) $\quad X=D_{4}^{\varepsilon}(q),\left.\quad V\right|_{X}=V_{X}\left(\lambda_{1}+\lambda_{3}\right), p \neq 2$,

$$
\begin{aligned}
& V_{X}\left(\lambda_{1}+\lambda_{3}\right) / V_{X}\left(\lambda_{1}\right), p=2, \\
& V_{X}\left(\lambda_{2}\right)^{2}, p \neq 2, \\
& V_{X}\left(\lambda_{1}\right)^{7} .
\end{aligned}
$$

We shall rule out all but a few cases by considering the possible dimensions of $C_{V}(t)$ for suitable semisimple elements $t$, and comparing these with Lemma 1.12.

First let $X=A_{4}^{\epsilon}(q)$. Define $t_{1}, t_{2}, t_{3} \in X$ with actions on the natural 5dimensional module as follows:

$$
\begin{aligned}
& p \neq 2: \quad t_{1}=\operatorname{diag}(-1,-1,1,1,1), t_{2}=\operatorname{diag}(-1,-1,-1,-1,1) \\
& p=2: \quad t_{3} \text { has order } 3 \text {, with exactly three eigenvalues } 1 .
\end{aligned}
$$

Then

$C_{V_{X}\left(\lambda_{1}\right)}\left(t_{i}\right)$ has dimension $3,1,3$ as $i=1,2,3$,

$C_{V_{X}\left(\lambda_{1}+\lambda_{4}\right)}\left(t_{i}\right)$ has dimension 12,16 as $i=1,2$ (or 11,15 if $p=5$ ),

$C_{V_{X}\left(\lambda_{k}+p^{a} \lambda_{m}\right)}\left(t_{i}\right)$ has dimension $13,17,11$ as $i=1,2,3$ for $k, m \in\{1,4\}, a>0$.

For each of the configurations in (i), we find from this that $C_{V}(t)$ does not have one of the dimensions given in Lemma 1.12.

For $X=C_{4}(q)$, define $t_{1}, t_{2} \in X$ with actions on $V_{X}\left(\lambda_{1}\right)$ as follows:

$$
\begin{aligned}
& p \neq 2: \quad t_{1}=\operatorname{diag}(-1,-1,1,1,1,1,1,1) \\
& p=2: \quad t_{2} \text { has order } 3, \text { with six eigenvalues } 1 .
\end{aligned}
$$

Then

$$
\begin{aligned}
& C_{V_{X}\left(\lambda_{1}\right)}\left(t_{i}\right) \text { has dimension } 6 \text { for } i=1,2, \\
& C_{V_{X}\left(\lambda_{3}\right)}\left(t_{i}\right) \text { has dimension } 20(p \neq 3), 14(p=3) \text { for } i=1,2 \text {, } \\
& C_{V_{X}\left(\lambda_{4}\right)}\left(t_{2}\right) \text { has dimension } 0(p=2) .
\end{aligned}
$$

Again, Lemma 1.12 gives a contradiction for all the configurations in (ii). 
Now suppose $X=D_{4}^{\varepsilon}(q)$. Pick a semisimple element $t \in X$ with action on $V_{X}\left(\lambda_{1}\right)$ of the form $\operatorname{diag}\left(1,1,1,1, \alpha, \alpha, \alpha^{-1}, \alpha^{-1}\right)$ for some $\alpha \in k^{*}$ of order 2 (if $p \neq 2$ ) or 3 (if $p=2$ ). Then $C_{V_{X}\left(\lambda_{1}\right)}(t)$ has dimension 4 and if $p \neq 2, C_{V_{X}\left(\lambda_{1}+\lambda_{3}\right)}(t)$ has dimension 28 (as $V_{X}\left(\lambda_{1}+\lambda_{3}\right)$ is a quotient of $V_{X}\left(\lambda_{1}\right) \otimes V_{X}\left(\lambda_{3}\right)$ by a submodule isomorphic to $\left.V_{X}\left(\lambda_{4}\right)\right)$. Together with Lemma 1.12, this shows that $\left.V\right|_{X}$ is not $V_{X}\left(\lambda_{1}+\lambda_{3}\right)$ nor is it seven copies of $V_{X}\left(\lambda_{1}\right)$. This leaves the possibilities $\left.V\right|_{X}=V_{X}\left(\lambda_{1}+\lambda_{3}\right) / V_{X}\left(\lambda_{1}\right), p=2$ or $V_{X}\left(\lambda_{2}\right) / V_{X}\left(\lambda_{2}\right), p \neq 2$.

When $p=2$ and $\left.V\right|_{X}=V_{X}\left(\lambda_{1}+\lambda_{3}\right) / V_{X}\left(\lambda_{1}\right)$, we let $u$ be a long root element in $X$, and hence an involution in $G$. We claim that $C_{V}(u)$ has dimension 34 or 38, contradicting Lemma 1.13. We first note that $\left.V\right|_{X}=V_{X}\left(\lambda_{1}+\lambda_{3}\right) \oplus V_{X}\left(\lambda_{1}\right)$, as $V$ is self-dual. We will view $u$ as an element in $\bar{X}$, an algebraic group of type $D_{4}$ acting on the module $V_{\bar{X}}\left(\lambda_{1}+\lambda_{3}\right) \oplus V_{\bar{X}}\left(\lambda_{1}\right)$. In particular, we may take $u$ to be $U_{\alpha_{1}}(1)$. On $V_{\bar{X}}\left(\lambda_{1}\right), u$ has a 6-dimensional fixed point space. Now, let $\bar{A}=\left\langle U_{ \pm \alpha_{1}}(t) \mid t \in k\right\rangle$ ( $k$ an algebraically closed field of characteristic 2 over which $\bar{X}$ is defined). For a fixed triple of integers $(a, b, c)$, set

$$
V_{(a, b, c)}=\sum\left\{V_{\bar{X}}\left(\lambda_{1}+\lambda_{3}\right)_{\mu} \mid \mu=\lambda_{1}+\lambda_{3}-x \alpha_{1}-a \alpha_{2}-b \alpha_{3}-c \alpha_{4} \text { for some } x \geqslant 0\right\} \text {. }
$$

Then $V_{(a, b, c)}$ is an $\bar{A}$-invariant subspace of $V_{\bar{X}}\left(\lambda_{1}+\lambda_{3}\right)$ and $\left.V_{\bar{X}}\left(\lambda_{1}+\lambda_{3}\right)\right|_{\bar{A}}=$ $\bigoplus V_{(a, b, c)}$. Using this decomposition, we find that it is straightforward to check that

$$
\left.V_{\bar{X}}\left(\lambda_{1}+\lambda_{3}\right)\right|_{\bar{A}}=\left(V_{2}\right)^{8} \oplus\left(V_{1}\right)^{8} \oplus\left(V_{4}\right)^{4} \oplus\left(V_{4}^{\prime}\right)^{2},
$$

where $V_{2}$ and $V_{1}$ are restricted irreducible $\bar{A}$-modules of dimension 2 and 1 , respectively, $V_{4}$ is a 4-dimensional $\bar{A}$-module with composition factors $V_{2}^{(2)} / V_{1} / V_{1}$, and $V_{4}^{\prime}$ is a 4-dimensional $\bar{A}$-module with composition factors $V_{2} / V_{2}$. Now by Corollary 3.9 of [2], $V_{4}^{\prime}$ is in fact a direct sum of two copies of $V_{2}$. So the only ambiguity lies in $V_{4}$, where $C_{V_{4}}(u)$ has dimension 2 or 3 . Thus $C_{V}(u)$ has dimension 34 or 38 as claimed.

Finally consider the case where $\left.V\right|_{X}=V_{X}\left(\lambda_{2}\right) / V_{X}\left(\lambda_{2}\right)$ and $p \neq 2$. Note that this action implies that $X$ does not lie in a proper parabolic of $G$. We will show that $X$ lies in a proper connected reductive subgroup of $G$, contradicting ( $\dagger$ ). For $p>7$ this follows from [32, Theorem 2]. So we assume $2<p \leqslant 7$.

Our argument is based upon identifying the $G$-conjugacy class of a root element $u \in X$. Let $S<X$ be a fundamental $\operatorname{SL}_{2}(p)$ containing $u$. Then $\left.V_{X}\left(\lambda_{2}\right)\right|_{S}=V_{S}(0)^{9} \oplus V_{S}(\lambda)^{8} \oplus V_{S}(2 \lambda)$, where $\lambda$ is the fundamental dominant weight for $S$. Moreover, by [2], we have that for $0 \leqslant a<p, 0 \leqslant b<p$, $\operatorname{dim}\left(\operatorname{Ext}_{s}^{1}(V(a \lambda), V(b \lambda))\right)=1$ if $a+b \in\{p-1, p-3\}$ and also $\{a, b\} \neq\{0, p-1\}$, and otherwise $\operatorname{dim}\left(\operatorname{Ext}_{S}^{1}(V(a \lambda), V(b \lambda))\right)=0$. We conclude that if $p=7$ the Jordan decomposition of $u$ acting on $V$ has at least eighteen $1 \times 1$ blocks, at least sixteen $2 \times 2$ blocks, and that no Jordan block has size larger than $6 \times 6$. Comparing this with Table 8 of Lemma 1.13 , we see that $u$ lies in the $2 A_{1}$-class of $G$.

Now suppose $p=5$. Then the Jordan decomposition of $u$ has at least fourteen $1 \times 1$ blocks. This observation implies that $u$ lies in one of the following $G$-conjugacy classes: $A_{1}, 2 A_{1},\left(3 A_{1}\right)^{\prime}, A_{2}$ or $A_{3}$.

For the case $p=3$, observe that by Lemma 1.9, $\left.V\right|_{X}=V_{X}\left(\lambda_{2}\right) \oplus V_{X}\left(\lambda_{2}\right)$. Hence $u$ has Jordan canonical form with eighteen $1 \times 1$ blocks, sixteen $2 \times 2$ blocks and two $3 \times 3$ blocks. Thus $u$ lies in the $2 A_{1}$ conjugacy class of $G$.

Now one checks that in each case, $u$ lies in an $A_{1}$-type subgroup of $G$ which acts completely reducibly on $V$ with composition factors having restricted high 
weights. (Note that the $\left(3 A_{1}\right)^{\prime}$-class is represented by $U_{\alpha_{2}}(1) U_{\alpha_{3}}(1) U_{\alpha_{5}}(1)$.) Now apply Lemma 1.14 to get a closed connected subgroup $U$ of $G$ which stabilizes $W$, a 28-dimensional $X$-invariant subspace of $V$. Moreover, the $\mathbb{F}_{p}$-points of $X, X(p)$, lie in the closed connected subgroup $\left\langle x^{-1} U x \mid x \in X\right\rangle$, which in turn lies in $\left(\operatorname{Stab}_{G}(W)\right)^{\circ}$. Since $X$ stabilizes $W$, we conclude that $X<\operatorname{Stab}_{G}(W)^{\circ}$. Now let $M$ be a maximal closed connected subgroup of $G$ containing $\operatorname{Stab}_{G}(W)^{\circ}$. Then $M$ either fixes a 28-dimensional subspace of $V$, or is irreducible on $V$; therefore $M$ is not parabolic, so $M$ is reductive. Now we have $X$ in a closed connected reductive subgroup of $G$, contrary to $(\dagger)$.

Lemma 3.7. Suppose $G=E_{7}, X$ has untwisted rank 4, and $X$ fixes a point on $V_{G}\left(\lambda_{7}\right)$. Then Theorem 2 holds. Moreover, if $X \neq D_{4}^{\varepsilon}(q)$, then long root subgroups of $X$ lie in long root subgroups of $G$.

Proof. Since point-stabilizers in $G$ have positive dimension, $X$ lies in a parabolic subgroup of $G$, and hence we see that $X$ lies in an $E_{6}$ or $D_{6}$ parabolic $P=Q L$. Inductively, we know the embedding of $X Q / Q$ in $L^{\prime} Q / Q$, so the weights of $X$ occurring in $Q$ are as listed in Table 12 .

TABLE 12

\begin{tabular}{ll}
\hline \multicolumn{1}{c}{$X$} & Modules $M$ in $\left.Q\right|_{X}$ \\
\hline$A_{4}^{\varepsilon}(q)$ & $\lambda_{1}, \lambda_{2}$ \\
$B_{4}(q), p \neq 2$ & $\lambda_{1}, \lambda_{4}$ \\
$C_{4}(q)$, & $\lambda_{1}$ or $\lambda_{4}$ for $p=2, \lambda_{2}$ \\
$D_{4}^{\varepsilon}(q)$ & $\lambda_{1}, \lambda_{3}, \lambda_{4}, \lambda_{2}(p=2)$ \\
$F_{4}^{\varepsilon}(q)$ & $\lambda_{4}$ \\
\hline
\end{tabular}

By $(\dagger), H^{1}(X, M) \neq 0$ for some $M$. Using Lemma 1.8 and replacing $P$ by a minimal parabolic $Q L$ containing $X$ we are left with the configurations listed in Table 13.

TABLE 13

\begin{tabular}{lllll}
\hline & $X$ & $\lambda$ & $L^{\prime}$ & $p$ \\
\hline$(1)$ & $C_{4}(q)$ & $\lambda_{1}$ & $D_{5}$ & 2 \\
$(2)$ & $C_{4}(q)$ & $\lambda_{2}$ & $E_{6}$ & 2 \\
$(3)$ & $D_{4}^{\varepsilon}(q)$ & $\lambda_{2}$ & $E_{6}$ & 2 \\
$(4)$ & $F_{4}(q)$ & $\lambda_{4}$ & $E_{6}$ & 3 \\
\hline
\end{tabular}

In cases (1) and (4), Lemma 1.8 gives that $H^{1}\left(X, V_{X}(\lambda)\right)$ has dimension 1 , and so by Lemma $1.2, H^{1}(X, Q)=0$ (as $Q$ is an indecomposable extension of $V_{X}(\lambda)$ ). Therefore $X$ lies in a conjugate of $L^{\prime}$, contrary to $(\dagger)$.

For case (2), argue as in Lemma 2.7 that there are just two classes of subgroups $C_{4}(q)$ in this case, each lying in a connected subgroup $C_{4}$. 
For case (3), refer to the end of the proof of Lemma 3.1 (case (9)) where we showed that $X$ lies in a subgroup $\bar{X}=D_{4}$; so (I) of Theorem 2 holds.

The last sentence of the lemma follows by inspection.

Lemma 3.8. Theorem 2 holds if $G=E_{8}$ and $X$ has untwisted rank 5. Moreover, in case (I) of Theorem 2, the long root subgroups of $X$ are contained in long root subgroups of $G$.

Proof. First assume that $X$ is one of $A_{5}(q), B_{5}(q)$ and $D_{5}(q)$. Let $A$ be a Levi subgroup $A_{4}(q)$ of $X$; then $A$ contains long root subgroups of $X$. Now $A$ is contained in a parabolic subgroup of $G$, and we choose $Q L$ to be a minimal such parabolic. We consider $\bar{A}=A Q / Q$ as a subgroup of $L$. It follows from the previous three lemmas that $L^{\prime}$ is classical. Then, applying Lemma 1.11 to the action of $\bar{A}$ on the natural module of $L^{\prime}$, we deduce that $L^{\prime}$ is in fact $A_{4}$. Now the weights of $L^{\prime}$ on the factors of $Q$ are $\lambda_{1}$ and $\lambda_{2}$ (cf. Lemma 1.5), and it follows from Lemma 1.8 that $A$ is contained in a conjugate of $L$. Hence the long root subgroups of $X$ are contained in long root subgroups of $G$, and the assertion follows from [25].

Now assume that $X$ is one of the remaining groups $C_{5}(q),{ }^{2} A_{5}(q)$ and ${ }^{2} D_{5}(q)$ and $X \neq{ }^{2} A_{5}(5)$ or ${ }^{2} D_{5}(3)$ (which are in (III) of Theorem 2). Choose a semisimple element $x$ in $X$ such that $C_{X}(x)$ normalizes a subgroup $C=C_{4}(q)$ in the first case and $C={ }^{2} A_{4}(q)$ in the second and third. If $C_{G}(x)$ has rank at most 7 , the result follows from previous cases. If $C=C_{4}(q)$ then $C$ centralizes a semisimple element of order greater than 3 , so $C_{G}(x)$ does indeed have rank at most 7 (see $[15,14.1]$ ). It remains to consider the case $C={ }^{2} A_{4}(q)$ with $C_{G}(x)$ a subgroup $A_{8}$ or $D_{8}$. The natural module $W$ for $C_{G}(x)$ either has dimension 9 , or is self-dual of dimension 16. It now follows from Lemmas 1.11 and 1.8 that $C$ is contained in a Levi subgroup of $C_{G}(x)$ of type $A_{4}$. The conclusion now follows as before: the long root subgroups of $C$ and hence of $X$ are contained in long root subgroups of $G$, so [25] gives the result.

LEMMA 3.9. Theorem 2 holds for $G=E_{6}, E_{7}$ or $E_{8}$.

Proof. By Lemmas 3.5-3.8, we may assume that $X$ has untwisted rank strictly greater than 4. Pick $Y$ a subgroup of $X$, containing a long root subgroup of $X$, such that

(i) if $G$ is $E_{6}$ or $E_{7}$ then $Y$ has rank 4 but $Y \neq D_{4}^{\varepsilon}(q)$,

(ii) if $G$ is $E_{8}$ then $Y$ has rank 5 .

By Lemmas 3.5-3.8, the long root subgroups of $Y$ are contained in long root subgroups of $G$. The claim now follows from [25].

Lemma 3.10. Theorem 2 holds if $G=G_{2}$.

Proof. Here $X$ has untwisted rank 2. If $X=G_{2}^{\varepsilon}(q)$, the result follows from [25, 5.1]. If $X$ stabilizes a 1 -space in $V_{G}\left(\lambda_{1}\right)$, then by $(\dagger), X$ lies in a parabolic of $G$, which is impossible. Hence $X \neq B_{2}^{\varepsilon}(q)$ (the only irreducible representations of $B_{2}^{\varepsilon}(q)$ of dimension at most 8 have dimensions 1,4 and 5). 
Now consider $X=A_{2}^{\varepsilon}(q)$. As $X$ does not stabilize a 1-space, $p=2$ or 3. If $X$ is reducible on $V_{G}\left(\lambda_{1}\right)$, then $X$ stabilizes a 3-space $W$, and the stabilizer of $W$ in $G$ has positive dimension, and hence is $A_{2}$, contrary to (†).

It remains to deal with the case where $X=A_{2}^{\varepsilon}(q)$ is irreducible on $V_{G}\left(\lambda_{1}\right)$. Since $X$ has an irreducible 7-dimensional representation only when the characteristic is 3 , we have $p=3$. Regard $G$ as $C_{D}(\tau)$, where $D=D_{4}$ and $\tau$ is a triality automorphism of $D$, and let $Y$ be a subgroup $A_{2}^{\varepsilon}(q)$ of $G$ containing short root elements of $G$. Then $Y=X^{g}$ for some $g \in D$, which implies that $\left\langle\tau, \tau^{g}\right\rangle$ centralizes $Y$, so $[g, \tau] \in C_{D}(Y)=1$, Therefore $g \in C_{D}(\tau)=G$, and so $X$ is $G$-conjugate to $Y$. Applying a graph morphism of $G$ (as in [36, Theorem 28]), we see that the conclusion follows from [25].

\section{Proof of Theorem 3}

Let $L=O^{p^{\prime}}\left(G_{\sigma}\right)$ be a finite exceptional simple group of Lie type, let $L_{1}$ be an almost simple group with socle $L$, and let $M$ be an almost simple maximal subgroup of $L_{1}$ with socle $X=X(q)$ satisfying the hypotheses of Theorem 3 . Observe that all automorphisms of $L$, and hence all elements of $L_{1}$, lift to automorphisms of $G$ (as abstract group) which commute with $\sigma$.

By Theorem 2, there is a simple closed connected $\sigma$-stable subgroup $\bar{X}$ of $G$ such that $X=O^{p^{\prime}}\left(\bar{X}_{\delta}\right)$ for some Frobenius morphism $\delta$ of $\bar{X}$.

We claim that $M$ normalizes $\bar{X}$. This follows from the proof of Lemma 3.1 (see ( $\ddagger$ and the discussion following it). If $\bar{X}=G$ then $X$ is a subfield or twisted subgroup, so assume that $\bar{X}<G$. Then $M=N_{L_{1}}(\bar{X})$, so $M$ contains $\left(\bar{X}_{\sigma}\right)^{\prime}$, and hence $X=\left(\bar{X}_{\sigma}\right)^{\prime}$.

By Theorem 1, there is a subsystem subgroup $Y$ of $G$ containing $\bar{X}$ such that one of conclusions (I)-(IV) of Theorem 1 holds. We consider each possibility in turn.

In case (IV), $C_{G}(\bar{X})$ contains a $\sigma$-stable unipotent subgroup $U$, and $1 \neq U_{\sigma} \leqslant$ $C_{L_{1}}(X)$, which is a contradiction as $M=N_{L_{1}}(X)$ is almost simple.

In case (III) we have $p=2$ and $\bar{X}<F_{4}<Y=E_{6}$; it follows from the proof of Lemma 3.1 that $\sigma$ and $N_{L_{1}}(\bar{X})$ normalize the subgroup $F_{4}$, which is impossible by the maximality of $M$.

In case (I), $\bar{X}=Y$, from which it follows that $M$ is a subgroup of maximal rank.

We are left with case (II), in which $\bar{X}=Y_{\tau}$ for some graph automorphism $\tau$ of $Y$. If $Y=G$ then $G=E_{6}$ and (iii) of Theorem 3 holds, so suppose that $Y \neq G$. Since $C_{L}(X)=1, C_{G}(\bar{X})^{0}$ must be either 1 or a non-trivial torus $T$. In the latter case $M=N_{L_{1}}(T)$ is a subgroup of maximal rank. Thus we may assume that $C_{G}(\bar{X})^{0}=C_{G}(Y)^{0}=1$. This forces $Y$ to have rank equal to that of $G$; a quick check shows that the possibilities are as follows: $G=E_{8}, Y=A_{8}$ or $D_{8} ; G=E_{7}$, $Y=A_{7} ; G=F_{4}, Y=D_{4}$; and $G=G_{2}, Y=A_{2}$.

The only case in which $N_{L_{1}}(\bar{X})$ does not normalize $Y$ occurs when $G=E_{7}$, $Y=A_{7}$ and $\bar{X}=D_{4}$ (in this case $G$ induces a full group $S_{3}$ of graph automorphisms on $\bar{X}$, see [13]). When $p=2$ the subgroup $\bar{X}$ lies in a subgroup $C_{4}$ of $Y$, and $C_{L}\left(C_{4}\right) \neq 1$ by $[25, \S 4]$. And when $p \neq 2, C_{L}(\bar{X}) \cong 2^{2}$ by $[13]$. Hence none of these cases can occur.

This completes the proof of Theorem 3 . 


\section{References}

1. J. Alperin and D. Gorenstein, 'A vanishing theorem for cohomology', Proc. Amer. Math. Soc. 32 (1972) 87-88.

2. H. H. Andersen, J. Jorgensen, and P. Landrock, 'The projective indecomposable modules of SL $\left(2, p^{n}\right)^{\prime}$, Proc. London Math. Soc. (3) 46 (1983) 38-52.

3. M. AsChBACHER and G. M. SEITZ, 'Involutions in Chevalley groups over fields of even order', Nagoya Math. J. 63 (1976) 1-91.

4. H. AZAD, M. BARRY, and G. M. SeItz, 'On the structure of parabolic subgroups', Comm. Algebra 18 (1990) 551-562.

5. P. BALA and R. W. CARTER, 'Classes of unipotent elements in simple algebraic groups II', Math. Proc. Cambridge Philos. Soc. 80 (1976) 1-17.

6. A. Borel and J. DE SEINBENTHAL, 'Les sous-groupes fermés de rang maximum des groupes de Lie clos', Comment. Math. Helv. 23 (1949) 200-221.

7. A. BOREL and J. TiTs, 'Éléments unipotents et sousgroupes paraboliques de groupes réductifs', Invent. Math. 12 (1971) 95-104.

8. N. BURGOYNE and C. WILliamson, 'Some computations involving simple Lie algebras', Proceedings of the 2nd Symposium on Symbolic and Algebraic Manipulation (ed. S. P. Petrick, New York Association of Computing Machinery, 1971), pp. 162-171.

9. M. CABANES, 'A criterion for complete reducibility and some applications', Astérisque (1993) 181-187.

10. R. W. CARTER, 'Centralizers of semisimple elements in finite groups of Lie type', Proc. London. Math. Soc. (3) 37 (1978) 491-507.

11. R. W. CARTER, Finite groups of Lie type: conjugacy classes and complex characters (WileyInterscience, New York, 1985).

12. E. Cline, B. Parshall, L. SCotT, and W. van der Kallen, 'Rational and generic cohomology', Invent. Math. 39 (1977) 143-163.

13. A. M. Cohen, M. W. Liebeck, J. Saxl, and G. M. Seitz, 'The local maximal subgroups of exceptional groups of Lie type, finite and algebraic', Proc. London. Math. Soc. (3) 64 (1992) $21-48$.

14. D. Deriziotis and M. W. LiebeCK, 'Centralizers of semisimple elements in finite twisted groups of Lie type', J. London Math. Soc. (2) 31 (1985) 48-54.

15. D. GORENSTEIN and R. LYONS, 'The local structure of finite groups of characteristic 2 type', Mem. Amer. Math. Soc. 276 (1983).

16. N. IWAHORI, 'Centralizers of involutions in finite Chevalley groups', Seminar on algebraic groups and related finite groups (eds A. Borel et al.), Lecture Notes in Mathematics 131 (Springer, Berlin, 1970), pp. 267-295.

17. J. C. JANTZEN, Representations of algebraic groups (Academic Press, Boston, 1987).

18. W. JONES and B. PARSHALL, 'On the 1-cohomology of finite groups of Lie type', Proceedings of a Conference on Finite Groups (eds W. Scott and F. Gross, Academic Press, New York, 1976).

19. A. S. KLeSHCHEv, 'Cohomology of finite Chevalley groups with coefficients in modules with 1-dimensional weight spaces', Comm. Algebra 22 (1994) 1197-1218.

20. R. LAWTHER, 'Jordan block sizes of unipotent elements in exceptional algebraic groups', Comm. Algebra, to appear.

21. M. W. LiebeCK, 'The affine permutation groups of rank three', Proc. London Math. Soc. (3) 54 (1987) $477-516$.

22. M. W. LIEBECK and J. SAXL, 'On the orders of maximal subgroups of the finite exceptional groups of Lie type', Proc. London Math. Soc. (3) 55 (1987) 299-330.

23. M. W. LIEBECK, J. SAXL, and G. M. SEITZ, 'Subgroups of maximal rank in finite exceptional groups of Lie type', Proc. London Math. Soc. (3) 65 (1992) 297-325.

24. M. W. LIEBECK and G. M. SEITZ, 'Maximal subgroups of exceptional groups of Lie type, finite and algebraic', Geom. Dedicata 36 (1990) 353-387.

25. M. W. LiEBECK and G. M. SEITZ, 'Subgroups generated by root elements in groups of Lie type', Ann. of Math. 139 (1994) 293-361.

26. M. W. LIEBECK and G. M. SEITZ, 'Reductive subgroups of exceptional algebraic groups', Mem. Amer. Math. Soc. to appear.

27. K. Mizuno, "The conjugate classes of unipotent elements of the Chevalley groups $E_{7}$ and $E_{8}$, Tokyo J. Math. 3 (1980) 391-461.

28. R. REE, 'A family of simple groups associated with a simple Lie algebra of type $\left(F_{4}\right)$ ', Amer. $J$. Math. 83 (1961) 401-420.

29. G. M. SEITZ, 'Generation of finite groups of Lie type', Trans. Amer. Math. Soc. 271 (1982) 351-407.

30. G. M. SEITZ, 'Maximal subgroups of exceptional algebraic groups', Mem. Amer. Math. Soc. 365 (1987).

31. G. M. SEITZ, 'Maximal subgroups of exceptional algebraic groups', Mem. Amer. Math. Soc. 441 (1991). 
32. G. M. SEITZ and D. M. TESTERMAN, 'Extending morphisms from finite to algebraic groups', J. Algebra 131 (1990) 559-574.

33. J.-P. SERRE, 'Sur la semi-simplicité des produits tensoriels de représentations de groupes', Invent. Math. 116 (1994) 513-530.

34. P. SiN, 'Extensions of simple modules for $\mathrm{Sp}_{4}\left(2^{n}\right)$ and $\mathrm{Suz}\left(2^{m}\right)$ ', Bull. London Math. Soc. 24 (1992) 159-164.

35. P. SIN, 'On the modular representations of the Hall-Janko group', preprint, University of Florida, Gainsville, 1994.

36. R. STEINBERG, Lectures on Chevalley groups, notes by J. Faulkner and R. Wilson, Yale University, 1968.

37. D. M. TESTERMAN, 'Irreducible subgroups of exceptional algebraic groups', Mem. Amer. Math. Soc. 390 (1988).

38. H. VOLKLEIN, 'On extensions between simple modules of a Chevalley group', Proceedings of the 2nd International Group Theory Conference (Brixen 1989), Rend. Circ. Math. Palermo 23 (1990) 337-346.

\section{Department of Mathematics \\ Imperial College \\ Huxley Building \\ 180 Queen's Gate \\ London SW7 2BZ}

\author{
Department of Pure Mathematics \\ and Mathematical Statistics \\ 16 Mill Lane \\ Cambridge $C B 21 S B$
}

Mathematics Institute

University of Warwick

Coventry CV4 $7 A L$ 